\title{
ATTITUDE DETERMINATION OF A STUDENT SATELLITE USING ON-ORBIT MAGNETOMETER AND TEMPERATURE DATA
}

\author{
A Thesis \\ presented to \\ the Faculty of \\ California Polytechnic State University \\ San Luis Obispo \\ In Partial Fulfillment \\ of the Requirements for the Degree \\ Master of Science in Aerospace Engineering
}

By

Jacob Daniel DiMiceli

June 2009 
(C)2009

Jacob Daniel DiMiceli

ALL RIGHTS RESERVED 


\section{APPROVAL PAGE}

TITLE: $\quad$ Attitude Determination of a Student Satellite Using On-Orbit

Magnetometer and Temperature Data

AUTHOR: Jacob DiMiceli

DATE SUBMITTED: 06/05/09

COMMITTEE MEMBER: $\quad$ Dr. Jordi Puig Suari

Advisor

COMMITTEE MEMBER: Dr. Eric Mehiel

Committee Member

COMMITTEE MEMBER: Dr. Kira Abercromby Committee Member

COMMITTEE MEMBER: Dr. Jeff Fisher

Committee Member 


\section{ABSTRACT \\ ATTITUDE DETERMINATION OF A STUDENT SATELLITE USING ON-ORBIT MAGNETOMETER AND TEMPERATURE DATA}

Jacob DiMiceli

An extended Kalman filter is modified to support only magnetometer data as a measurement for spacecraft attitude information. Actual on-orbit data from a student satellite is used in the filter from three different time periods and varying data frequencies. The extended Kalman filter has a thermal model that utilizes external temperature data as a verification tool for filter convergence and for parameter tuning. It can capture the frequency of temperature changes and the correct side panel being lit by the sun as long as the filter converges properly, but not the magnitude of the temperature at a specific time. A solar panel power model is also attempted, which was found to be unusable due to power budget issues and the side panel current sensors railing. Convergence of the three sets of data showed that a 30 second snapshot rate converges to a more accurate attitude information when the satellite has a $0.1 \mathrm{rev} / \mathrm{min}$ rotation rate compared to the $1.0 \mathrm{rev} / \mathrm{min}$ rotation rate data. A 10 second snapshot rate gives more accurate attitude information for data from a satellite with a $1.0 \mathrm{rev} / \mathrm{min}$ rotation rate. By investigating the drastic reduction in the satellite's rotation rate over 6 months, it was discovered that the satellite's antenna is most likely magnetized, causing it to try to align itself with the Earth's magnetic field, slowing down the rotation rate of the satellite from $1 \mathrm{rev} / \mathrm{min}$ to $0.1 \mathrm{rev} / \mathrm{min}$. 


\section{ACKNOWLEDGEMENTS}

I would like to thank my parents for always being there for me and pushing me to excel at everything that I do. Laurel, thank you for being supportive especially during the hard times, you kept me strong when I needed you the most. Thanks to everyone in Polysat who made going into the lab a great experience, even if it was a late night satellite pass. Dr. P, thank you for getting me interested in Polysat and giving me the opportunity to be part of something great. Jeff, thanks for helping through the tough decisions during the final days of this thesis. 


\section{TABLE OF CONTENTS}

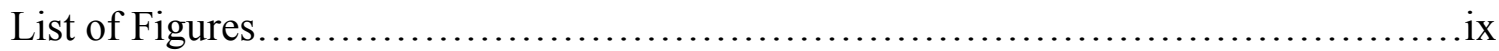

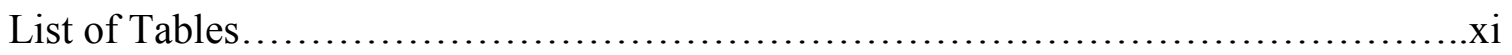

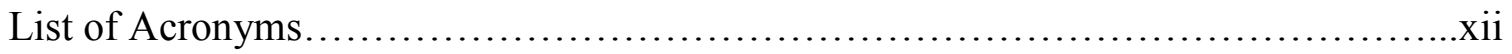

List of Symbols.................................................................

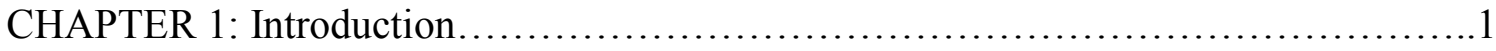

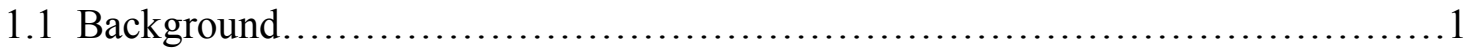

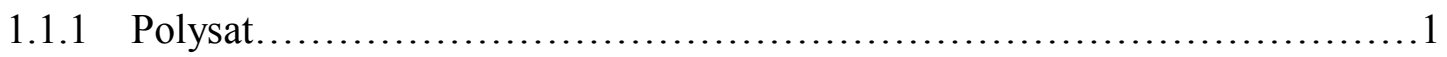

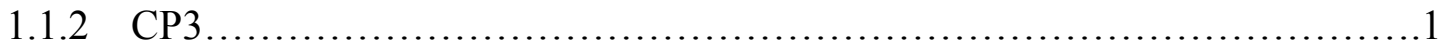

1.1.3 Satellite Operations..................................................

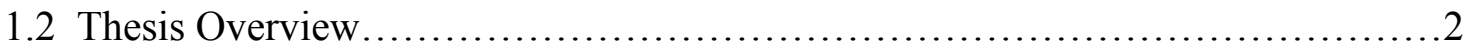

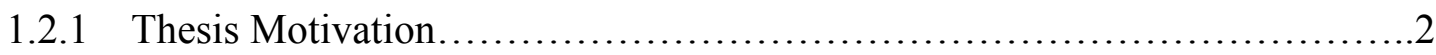

1.2.2 Thesis Framework.....................................................

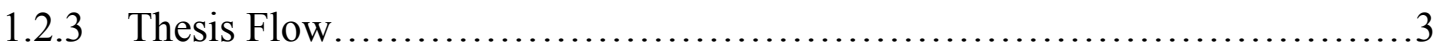

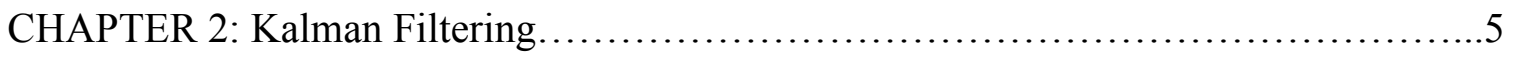

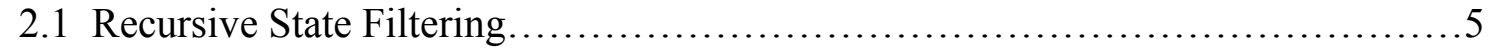

2.2 Extended Kalman Filter....................................................... 5

2.2.1 Linear State Space Model.............................................6

2.2.2 Kalman Filter Equations.............................................

2.2.3 Nonlinear Kalman Filter Revisions...................................... 8

CHAPTER 3: Polysat Extended Kalman Filter.......................................10

3.1 Reference Frames....................................................... 10

3.1.1 Reference Frame Definitions........................................10 
3.1.2 Frame Transformations...............................................

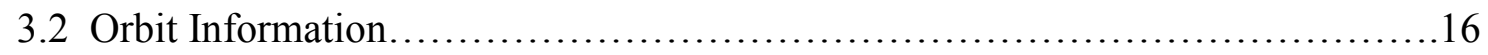

3.2.1 Satellite Took Kit....................................................

3.2.2 Universal Time Conversion..............................................16

3.3 Reference Vector Definition................................................... 17

3.3.1 Magnetic Inertial Reference Vector....................................17

3.3.2 Solar Inertial Reference Vector....................................... 17

3.3.3 Cubesat Inertial Reference Vector......................................19

3.4 Sensor Calibration........................................................... 19

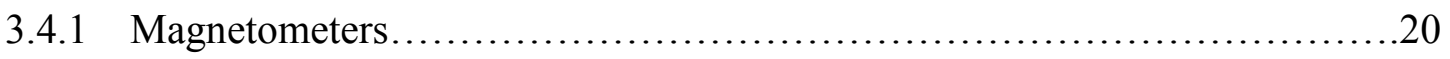

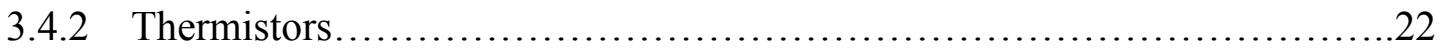

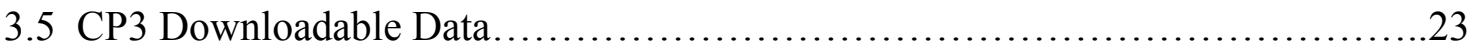

3.5.1 Programmed Data Packages...........................................23

3.5.2 Mitigating Dropped Packet Effects....................................23

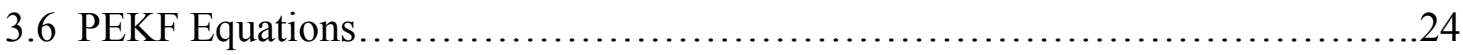

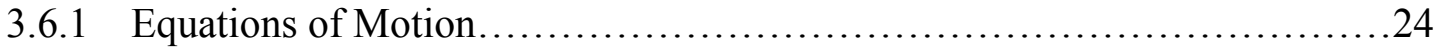

3.6.2 Reduced State and Measurement Equations...............................25

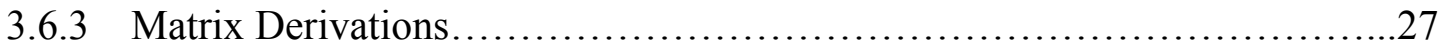

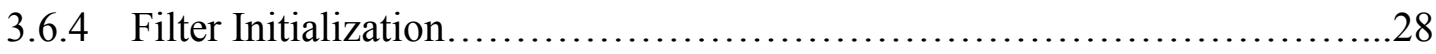

CHAPTER 4: Satellite Thermal Model..........................................29

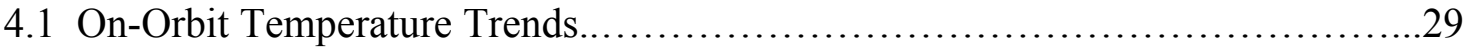

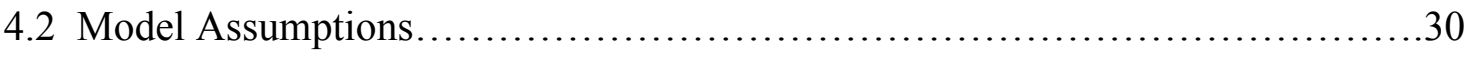

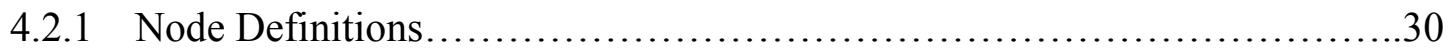


4.2.2 Node Heat Transfer Connections....................................... 32

4.2.3 Equation Derivations................................................

4.2.4 Using the Thermal Model for Verification............................... 37

4.3 Filter Simulation.............................................................

4.3.1 Satellite Emulator............................................... 37

4.3.2 Emulated Filter Performance..........................................38

CHAPTER 5: PEKF Performance with CP3 Data................................ 39

5.1 Filter Results.......................................................... 39

5.2 Filter Convergence...........................................................

5.2.1 July $9^{\text {th }}, 2007$ Data................................................ 40

5.2.2 July $12^{\text {th }}, 2007$ Data..............................................43

5.2.3 February $28^{\text {th }}, 2008$ Data........................................ 45

5.3 Solar Panel Current Issues.............................................47

5.4 Rotation Rates........................................................49

5.5 Magnetic Field Alignment............................................51

CHAPTER 6: Conclusion....................................................53

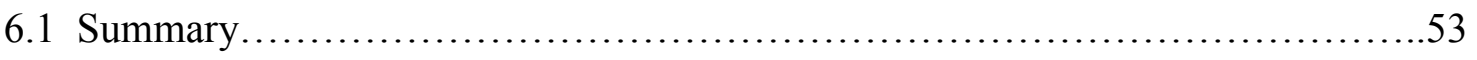

6.2 Recommendation for Future Work ....................................... 54

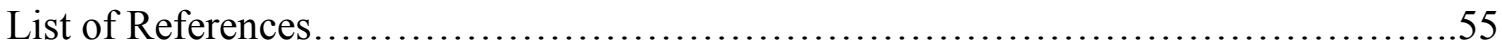




\section{LIST OF FIGURES}

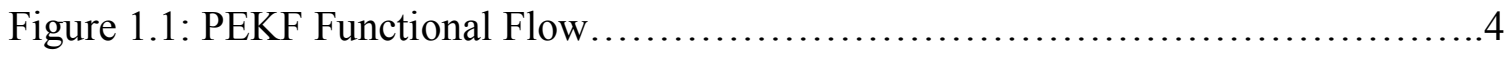

Figure 3.1: Reference Frames Related to the Center of the Earth.................... 10

Figure 3.2: Geodetic Latitude Representation..................................12

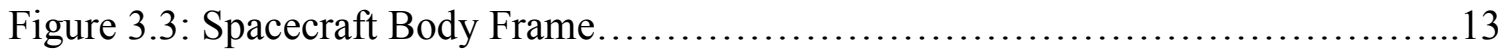

Figure 3.4: Neighboring Frames.......................................... 14

Figure 3.5: CP3 Side Panel Sensors.........................................20

Figure 3.6: Side Panel Magnetometer Calibration...............................21

Figure 3.7: Magnetometer Calibration Excel Tool.............................22

Figure 4.1: Side Panel Temperature Data for June $18^{\text {th }}, 2007 \ldots \ldots \ldots \ldots \ldots \ldots \ldots \ldots \ldots . . .29$

Figure 4.2: Side Panel Temperature Data for November $18^{\text {th }}, 2007 \ldots \ldots \ldots \ldots \ldots \ldots \ldots . \ldots . \ldots . \ldots$

Figure 4.3: Thermal Node Connections..................................... 32

Figure 4.4: Shape Factor Equations for External Nodes............................ 37

Figure 4.5: PEKF Simulation for Filter Testing............................... 38

Figure 5.1: July $9^{\text {th }}, 2007$ Magnetic Field Vector Norms...........................41

Figure 5.2: July $9^{\text {th }}, 2007$ Side Panel Temperatures...............................41

Figure 5.3 July $9^{\text {th }}, 2007$ Side Panel Temperature Error............................42

Figure 5.4: July $12^{\text {th }}, 2007$ Magnetic Field Vector Norms..........................43

Figure 5.5: July $12^{\text {th }}, 2007$ Side Panel Temperatures.............................44

Figure 5.6 July $12^{\text {th }}, 2007$ Side Panel Temperature Error..........................44

Figure 5.7: February $28^{\text {th }}, 2008$ Magnetic Field Vector Norms........................45

Figure 5.8: February $28^{\text {th }}, 2008$ Side Panel Temperatures.........................46

Figure 5.9. February $28^{\text {th }}, 2008$ Side Panel Temperature Error.......................47 
Figure 5.10: February $28^{\text {th }}, 2008$ Normalized Solar Panel Power........................48

Figure 5.11: July $9^{\text {th }}, 2007$ Rotation Rates.....................................49

Figure 5.12: July $12^{\text {th }}, 2007$ Rotation Rates...................................50

Figure 5.13: February $28^{\text {th }}, 2008$ Rotation Rates...................................

Figure 5.14: Magnetic Field Vectors in the Body Frame............................52 


\section{LIST OF TABLES}

Table 4.1: Thermal Model Constants............................................. 31 


\section{LIST OF ACRONYMS}

$\begin{aligned} \text { ADC } & \text { Attitude Determination and Control } \\ \text { CIRV } & \text { Cubesat Inertial Reference Vector } \\ \text { C\&DH } & \text { Command and Data Handling } \\ \text { CBEV } & \text { Cubesate Body Estimated Vector } \\ \text { ECEF } & \text { Earth-Centered Earth-Fixed } \\ \text { ECI } & \text { Earth-Centered Inertial } \\ \text { EKF } & \text { Extended Kalman Filter } \\ \text { GMST } & \text { Greenwich Mean Sidereal Time } \\ \text { IGRF-10 } & 10^{\text {th }} \text { Generation International Geomagnetic Reference Field } \\ \text { IR } & \text { Infrared Raditation } \\ \text { LLA } & \text { Latitude, Longitude, and Altitude } \\ \text { LNED } & \text { Local North East Down } \\ \text { MBRV } & \text { Magnetic Body Reference Vector } \\ \text { MIRV } & \text { Magnetic Inertial Reference Vector } \\ \text { PCB } & \text { Printed Circuit Board } \\ \text { PEKF } & \text { PolySat Extended Kalman Filter } \\ \text { P-POD } & \text { Poly-Picosatellite Orbital Deployer } \\ \text { PST } & \text { Pacific Standard Time } \\ \text { SBEV } & \text { Solar Body Estimated Vector } \\ \text { SIRV } & \text { Solar Inertial Reference Vector } \\ \text { STK } & \text { Satellite Tool Kit } \\ \text { TLE } & \text { Two Line Element } \\ \text { UT } & \text { Universal Time }\end{aligned}$




\section{LIST OF SYMBOLS}

Upper Case

A

$\mathrm{A}(\mathrm{)}$

B

$\mathrm{E}_{\mathrm{b}}$

$\mathrm{F}$

$\mathrm{F}$

$\mathrm{F}_{\mathrm{V}}$

$\mathrm{F}_{\mathrm{A}}$

$\mathrm{G}$

$\mathrm{G}_{\mathrm{o}}$

$\mathrm{H}$

I

J

$\mathrm{J}$

K

L

$\mathrm{P}$

$\mathrm{Q}$

$\dot{Q}$

$\mathrm{R}$

$\mathrm{T}$

$\mathrm{T}$

$\mathrm{T}_{\mathrm{s}}$

$\mathrm{V}$

W

Lower Case

a

b

c

c()

e

$\mathrm{f}$

$\mathrm{g}$

h ()

$\mathrm{h}$

k

1

n

$\mathrm{p}$
Definition

Surface Area

Quaternion Rotation Equation

Control Matrix

Total Emissive Power of a Black Body

Linear Dynamics Matrix

Shape Factor

Volume Composition Factor

Surface Area Composition Factor

Irradiation

Solar Constant

Linear Measurement Matrix

Identity Matrix

Moment of Inertia Matrix

Radiosity

Kalman Gain Matrix

Specific Angular Momentum

Covariance Matrix

Process Noise Matrix

Heat Transfer Rate

Measurement Noise Matrix

Temperature

Transformation Matrix

Sampling Time

Volume

Mean Longitude of the Sun

Semi Major Axis

Magnetic Field

Specific Heat Capacity

Cosine

Eccentricity

System Model

Mean Anomaly of the Sun

Measurement Equation

Altitude

Thermal Conductivity

Side Panel Thickness

Number of Materials in Thermal Node

Quaternion Unit Vector
Units

$\mathrm{m}^{2}$

$\mathrm{N} / \mathrm{A}$

$\mathrm{N} / \mathrm{A}$

$\mathrm{W} / \mathrm{m}^{2}$

$\mathrm{N} / \mathrm{A}$

N/A

$\mathrm{N} / \mathrm{A}$

$\mathrm{N} / \mathrm{A}$

$\mathrm{W} / \mathrm{m}^{2}$

$\mathrm{W} / \mathrm{m}^{2}$

$\mathrm{N} / \mathrm{A}$

$\mathrm{N} / \mathrm{A}$

$\mathrm{kg}-\mathrm{m}^{2}$

$\mathrm{W} / \mathrm{m}^{2}$

$\mathrm{N} / \mathrm{A}$

$\mathrm{km}^{2} / \mathrm{sec}$

$\mathrm{N} / \mathrm{A}$

$\mathrm{N} / \mathrm{A}$

$\mathrm{W}$

N/A

${ }^{\circ}$ Celsius

$\mathrm{N} / \mathrm{A}$

Seconds

$\mathrm{m}^{3}$

Degrees

$\mathrm{km}$

Gauss

$\mathrm{J} / \mathrm{kg}-\mathrm{K}$

N/A

N/A

N/A

Degrees

Gauss

$\mathrm{km}$

$\mathrm{W} / \mathrm{m}-\mathrm{K}$

$\mathrm{m}$

N/A

$\mathrm{km}$ 
Greek

$\omega$

$\mu$

Overscript

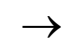

$\sim$

$-$

Superscript

Body

$\mathrm{T}$

$+$

$-$

Quaternion
Error Quaternion
Sine
Time
Control Input Vector
Measurment Noise
Process Noise
State Vector
Measurement Vector

Angular Rate

Right Ascension of the Sun

Quaternion Angle

Geodetic Latitude

Ecliptic Longitude of the Sun

Obliquity of the Ecliptic Plane of the Sun

Absorbtivity

Emissivity

Longitude

Sun Incidence Angle on Side Panel

State Transition Matix

A Change In

Density

Declination of the Sun

Standard Deviation

Stefan Boltzmann Constant
N/A

$\mathrm{N} / \mathrm{A}$

$\mathrm{N} / \mathrm{A}$

sec

N/A

N/A

N/A

$\mathrm{N} / \mathrm{A}$

N/A

$\mathrm{rad} / \mathrm{sec}$

Degrees

Radians or Degrees

Degrees

Degrees

Degrees

N/A

N/A

Degrees

Degrees

N/A

N/A

$\mathrm{kg} / \mathrm{m}^{3}$

Degrees

N/A

$\mathrm{W} / \mathrm{m}^{2}-\mathrm{K}^{4}$

$\begin{array}{cc}\text { Vector } & \text { N/A } \\ \text { Reduced State } & \text { N/A } \\ \text { Four Component Vector } & \text { N/A } \\ \text { Estimate } & \text { N/A }\end{array}$

Subscript

The $i^{\text {th }}$ element of the time vector

N/A

First Vector Component

N/A

In the Body Frame

$\mathrm{N} / \mathrm{A}$

A Posteriori

N/A

A Priori

N/A 


$\begin{array}{rcc}4 & \text { Fourth Vector Component } & \text { N/A } \\ \oplus & \text { Earth } & \text { N/A } \\ \text { SR } & \text { Solar Radiation } & \text { N/A } \\ \mathrm{A} & \text { Albedo and Earth IR } & \text { N/A } \\ \text { ER } & \text { Emitted Radiation } & \text { N/A } \\ \mathrm{C} & \text { Thermal Conduction } & \text { N/A } \\ \text { AR } & \text { Absorbed Radiation } & \text { N/A } \\ \text { int } & \text { Internal } & \text { N/A } \\ \text { ext } & \text { External } & \text { N/A } \\ \text { ei } & \text { External Affect on Internal } & \text { N/A }\end{array}$




\section{CHAPTER 1: INTRODUCTION}

\subsection{Background}

\subsubsection{Polysat}

Started by Dr. Jordi Puig Suari in 2000, Polysat is a student run satellite development project working out of Cal Poly in San Luis Obispo. The project focuses on student involvement in the life cycle of a satellite, from design to operation. The satellites designed by Polysat follow the Cubesat standard, which was developed in collaboration with Dr. Puig Suari and Stanford professor Dr. Robert Twiggs. The Cubesat standard limits satellites to a 10 -centimeter cube with a maximum mass of 1 kilogram. This standard allows student built satellites to gain access to launch opportunities through a satellite deployer developed by Polysat's sister project, Cubesat. This deployer, known as the P-POD, is an enclosed vessel capable of releasing three cubesats from a launch vehicle while ensuring the safety for other payloads onboard. By the end of 2009, Polysat will have designed and built four satellites and launched three of them, including one re-flight. The two Polysat satellites with the longest space heritage are known as CP3 and CP4.

\subsubsection{CP3}

CP3 was launched on a Dnepr rocket out of Kazakhstan on April 17 of 2007, marking one of the first Polysat satellites to be launched into space. It was placed in a 750 kilometer, sun-synchronized orbit with an inclination of 98 degrees. The BUS on CP3 includes the power, command and data handling $(\mathrm{C} \& \mathrm{DH})$, and communication systems for Polysat's satellites. The side panels on CP3 contain a suite of sensors that measure temperature, solar cell current, solar cell voltage, and the magnetic field using a two-axis 
Honeywell HMC1052 magnetometer. With five side panels on both satellites containing two-axis magnetometers, three-axis magnetic field information is available with redundancy in all axes.

\subsubsection{Satellite Operations}

Once first contact was made with CP3, a problem with the uplink was quickly discovered. This problem hindered the satellite's operation plans and limited its utility. Besides the flaws in the satellite's communication systems, the periodic nature of successful uplinks pointed towards an issue with its attitudes and rotation rates. By using many of the successful uplinks for data dumps from the sensors on the side panels and on the BUS, information about the satellite's health and status has been collected for most of its life in space. Utilizing this information is crucial to discovering the behavior of the satellite in space.

\subsection{Thesis Overview}

\subsubsection{Thesis Motivation}

This thesis is a continuation of work done by previous students in the Polysat project to develop a tool for determining attitude and rotation rates of satellites using on-orbit data. Erick Sturm developed an earlier version of this tool, called the Polysat Extended Kalman Filter (PEKF), prior to the launch of CP3. Sturm chose the Kalman filter for state estimation with the hope that the satellite would eventually be able to implement it on board for real time attitude determination. Sturm also saw the opportunity for a worthwhile learning exercise since the Kalman filter is a widely used state estimation technique. By continuing Erick's work, most of the filter development was already completed and set up specifically for CP3. The PEKF uses only magnetometer data as a 
measurement of the states with solar cell power as verification for filter convergence. It used a satellite emulator to simulate magnetometer and sun vector data in order to test and prepare the filter for use with on-orbit data.

\subsubsection{Thesis Framework}

The ultimate goal of this thesis is to obtain reasonable attitude information from the PEKF using verification techniques to ensure proper convergence of the filter. A secondary goal is to use this attitude information to confirm theories about CP3's dynamics from the raw on-orbit data. Raw magnetometer data suggested that the satellite's rotation rate reduced significantly over a 6-month period. The data also pointed towards a permanent magnet onboard the spacecraft, which could cause the rotation rate dampening. In order to obtain this goal, two verification techniques are explored using on-orbit side panel temperatures and solar panel currents. Both techniques involve a transformation of the inertial Sun vector using the states of the filter. This transformed Sun vector is manipulated for the comparison with on-orbit data.

\subsubsection{Thesis Flow}

This thesis will give an overview of the PEKF and data processing necessary to run the filter. This overview includes transformation matrices, PEKF equations, sensor calibrations, and reference vector creation. The side panel temperature verification technique will be developed using a thermal model of the satellite. Sturm already developed the solar panel solar panel verification technique leaving only the analysis of its performance. Three on-orbit data packages with different dates and frequency of the data will be used with the filter to accomplish the goals of the thesis. The functional flow of the PEKF is demonstrated in Figure 1.1. 


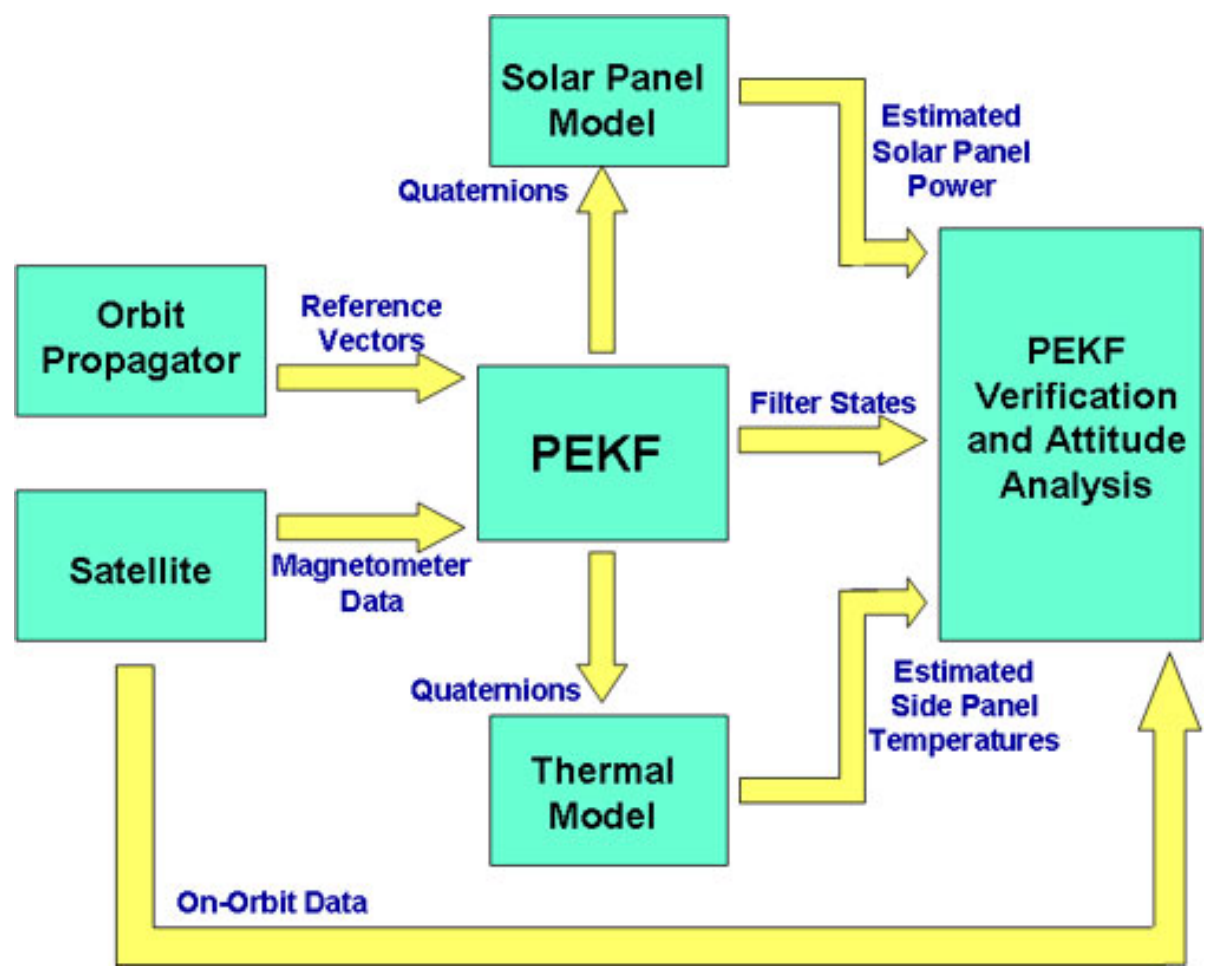

Figure 1.1. PEKF Functional Flow 


\section{CHAPTER 2: KALMAN FILTERING}

\subsection{Spacecraft State Estimation}

There are two commonly used techniques for state estimation of spacecraft: batch filtering and recursive state filtering. Batch filtering utilizes all of the available attitude measurements to update the state vector being used to represent the spacecraft's attitude. Batch filters are typically used during post-processing state estimation since all of the measurements are available. Recursive state filters can work in both real-time and postprocessing by using each measurement along with all previous measurements to update attitude information as the measurements are taken. As Wertz points out, the recursive state filters can be unstable due to their sensitivity to individual data points even though they can converge more quickly than batch filters (437). This paper will focus on a type of recursive state filter known as a Kalman filter since it is a continuation Sturm's Polysat Extended Kalman Filter.

\subsection{Extended Kalman Filter}

The Kalman filter was developed to determine the state of a linear system by minimizing the mean of the squared error using measured data and a relatively basic model of the system. The filter accomplishes this through a series of equations that predict and update the current state of the system through recursive processing of the measured data. Adaptations to the Kalman filter were discovered shortly after its creation, which allowed the filter to deal with nonlinear systems through a series of first order approximations. This new filter, called the Extended Kalman Filter (EKF), estimates the states of nonlinear systems using the Kalman filter equations with slight modifications. 


\subsubsection{Linear State Space Model}

The linear Kalman filter works to discover the states $(\vec{x})$ of a process that is modeled by a continuous linear state space equation of the form

$$
\dot{\vec{x}}=F \vec{x}+B \vec{u}+\vec{w},
$$

where the $F$ matrix links the state to its derivative, the $B$ matrix links the control input $(\vec{u})$ to the current state, and $\vec{w}$ is the random process noise. The measurements of the process $(\vec{z})$ are related to the states by

$$
\vec{z}=H \vec{x}+\vec{v},
$$

where $\mathrm{H}$ links the states to the measurements and $\vec{v}$ is the random measurement noise. By assuming there is no control input into the system and discretizing the equations, the state space and measurement equations become

$$
\begin{gathered}
\vec{x}_{i}=\Phi \vec{x}_{i-1}+\vec{w}_{i-1}, \\
\vec{z}_{i}=H \vec{x}_{i}+\vec{v}_{i},
\end{gathered}
$$

where the variable $i$ represents the current time step. The $\Phi$ matrix is known as the state transition matrix, which is used to propagate the discrete Ricatti equations. The state transition matrix is computed using the first two terms of Taylor series expansion of

$\left.e^{F t}\right|_{T_{s}}$ in order to retain the accuracy needed for the filter. The following equation is used to calculate the state transition matrix.

$$
\Phi\left(T_{s}\right)=I+F T_{s}
$$

The variable $T_{\mathrm{s}}$ represents the amount of time between measurements. 


\subsubsection{Kalman Filter Equations}

By letting $\hat{\vec{x}}_{i}^{-}$represent the a priori state estimate and $\hat{\vec{x}}_{i}^{+}$represent the a posteriori state estimate (indicated by the superscripted minus and plus signs), the $a$ priori and $a$ posteriori state error vectors can be defined as

$$
\begin{aligned}
& \Delta \vec{x}_{i}^{-}=\vec{x}_{i}-\hat{\vec{x}}_{i}^{-} \\
& \Delta \vec{x}_{i}^{+}=\vec{x}_{i}-\hat{\vec{x}}_{i}^{+}
\end{aligned}
$$

A caret above a vector or variable labels it as an estimate. These error vectors give rise to the a priori and a posteriori error covariance matrices, which are described by the equations below.

$$
\begin{aligned}
& P_{i}^{-}=E\left[\Delta \vec{x}_{i}^{-} \Delta \vec{x}_{i}^{-T}\right] \\
& P_{i}^{+}=E\left[\Delta \vec{x}_{i}^{+} \Delta \vec{x}_{i}^{+T}\right]
\end{aligned}
$$

The equation that minimizes the a posteriori error covariance matrix is known as the Kalman gain, which is represented by the following equation.

$$
K_{i}=P_{i}^{-} H^{T}\left(H P_{i}^{-} H^{T}+R\right)^{-1}
$$

The $R$ matrix in the Kalman gain equation is known as the measurement noise matrix. It is a diagonal matrix that contains the squared standard deviations, or variances, of the measurement sensors corresponding to each row. The $R$ matrix usually needs to be updated unless the measurement sensors are the same at each time step. The following equations update the state vector and covariance matrix.

$$
\begin{gathered}
\hat{\vec{x}}_{i}^{+}=\hat{\vec{x}}_{i}^{-}+K_{i}\left(\vec{z}-H \hat{\vec{x}}_{i}^{-}\right) \\
P_{i}^{+}=\left(I-K_{i} H_{i}\right) P_{i}^{-}
\end{gathered}
$$

The final equations of the filter propagate the state vector and covariance matrix. 


$$
\begin{gathered}
\hat{\vec{x}}_{i+1}^{-}=\Phi \hat{\vec{x}}_{i}^{+} \\
P_{i+1}^{-}=\Phi P_{i}^{+} \Phi^{T}+Q_{i}
\end{gathered}
$$

The $Q_{i}$ matrix is known as the process noise matrix, which is similar to the $R$ matrix in its relationship to the random noise vectors. While the $R$ matrix is the expected inner product of the measurement noise vector with itself, the $Q_{i}$ matrix is the expected inner product of the process noise vector with itself. It can be calculated with the following equation.

$$
Q_{i}=\int_{0}^{T_{s}} \Phi(t) Q \Phi^{T}(t) d t
$$

The process noise matrix will change at each time step when the state transition matrix does, as shown in the equation. The linear Kalman filter is broken down into a time update phase and a measurement update phase. The time update phase begins the filter process using the state vector and covariance matrix update equations along with initial estimates of the state and covariance matrix. The measurement update phase follows by calculating the Kalman gain, the propagated state vector, and the propagated covariance matrix propagation equations.

\subsubsection{Nonlinear Kalman Filter Revisions}

The nonlinear Kalman filter, or EKF, tackles the nonlinear state space model represented in the following equations without control inputs.

$$
\begin{aligned}
& \dot{\vec{x}}=f(\vec{x}, t)+\vec{w}(t) \\
& \vec{z}=h(\vec{x}, t)+\vec{v}(t)
\end{aligned}
$$

By representing the $F$ and $H$ matrices of the linear Kalman filter as first order approximations of the nonlinear state and measurement equations about the estimated 
state, the Kalman filter equations can be used. These approximations are shown in the subsequent equations.

$$
\begin{aligned}
& F_{i}=\left.\frac{d}{d \vec{x}} f\left(\vec{x}_{k}, t\right)\right|_{\hat{\hat{x}}_{k}^{+}} \\
& H_{k}=\left.\frac{d}{d \vec{x}} h\left(\vec{x}_{k}, t\right)\right|_{\hat{x}_{k}^{-}}
\end{aligned}
$$

As Sturm points out, this linearization is based on the assumption that the state estimate is close to the actual state (39). If this assumption breaks down, the filter will not converge. 


\section{CHAPTER 3: POLYSAT EXTENDED KALMAN FILTER}

\subsection{Reference Frame}

\subsubsection{Reference Frame Definitions}

The PEKF utilizes five reference frames to define measured and reference vectors. Three of the reference frames are related to the Earth's center, which are shown in Figure 3.1 (Sturm 7-9).
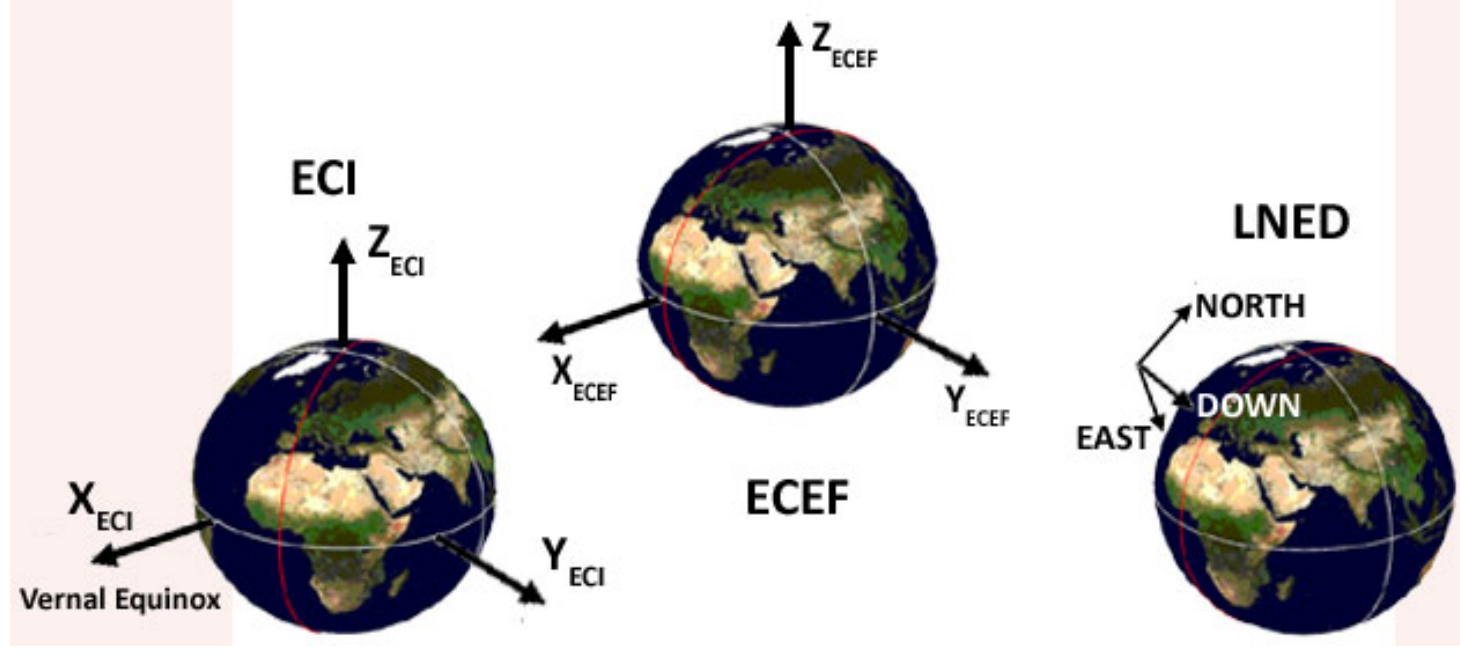

Figure 3.1. Reference Frames Related to the Center of the Earth

\subsubsection{Earth-Centered Inertial}

The Earth-Centered Inertial (ECI) frame is the first frame related to the center of the Earth. As the name suggests, it is unchanging in inertial space with its center at the center of the Earth. The x-axis points from the Earth to the Sun on the vernal equinox, the z-axis is aligned with the Earth's orbital angular momentum vector, and the y-axis completes the right-handed coordinate system. 


\subsubsection{Earth-Centered Earth-Fixed}

Another Earth-centered reference frame is the Earth-Centered Earth-Fixed (ECEF) frame. The ECEF frame shares z-axes with the ECI frame while the $\mathrm{x}$-axis and y-axis rotate with the Earth's rotation. The X-axis runs through the point on the Earth where the Prime Meridian intersects the Equator, and the y-axis finishes the right-handed coordinate system.

\subsubsection{Local North East Down}

The Local North East Down reference frame (LNED) refers to a nadir pointing coordinate system centered on a spacecraft. The other two axes point towards local East and local North.

\subsubsection{Geodetic}

The Geodetic reference frame models the Earth's surface as an ellipsoid and uses three parameters to describe the position of an object with respect to the Earth: latitude, longitude, and altitude (LLA). Geodetic latitude refers to the position of an object North and South of the equator. Since the Earth's surface is modeled as an ellipsoid, geodetic latitude is measured from the equatorial plane to a line that is normal to the surface of the Earth while intersecting the position of the object, as portrayed in Figure 3.2. It ranges 


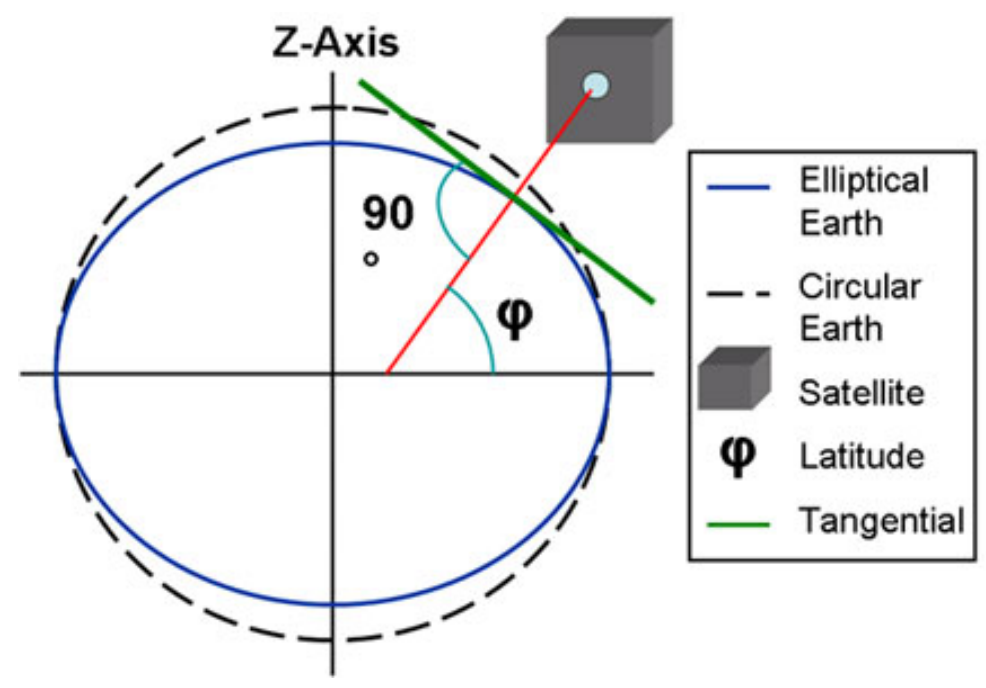

Figure 3.2. Geodetic Latitude Representation

between $-90^{\circ}$ at the South Pole to $90^{\circ}$ at the North Pole, with $0^{\circ}$ latitude lying at the equator. Longitude refers to the angle between the prime meridian and an object within the plane of the equator. It ranges between $-180^{\circ}$ west of the prime meridian to $180^{\circ}$ east of the prime meridian. Altitude is the distance between an object and the ellipsoidal surface of the Earth in a direction that is normal to the surface.

\subsubsection{Spacecraft Body}

The final reference frame is a right-handed coordinate system centered on the spacecraft called the spacecraft body frame. The axes are normal to three sides of the satellite, as shown in Figure 3.3. Two sides of note on the spacecraft are the negative y-axis, which contains the antenna for the satellite, and the z-axis, which contains the payload face. The payload face does not contain any solar cells or sensors. 


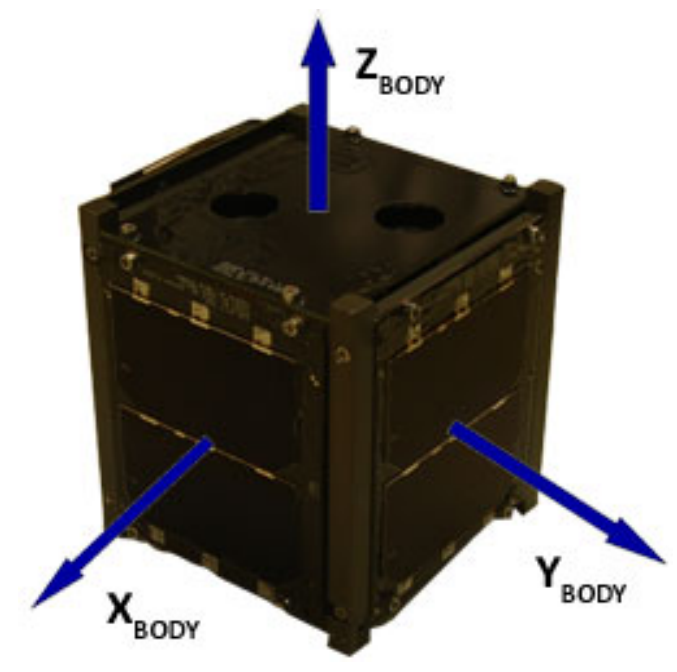

Figure 3.3. Spacecraft Body Frame

\subsubsection{Frame Transformations}

In order to stay consistent, the vectors used in the filter are transformed into the ECI frame with the ultimate goal of finding the relationship between the ECI frame and the spacecraft body frame. Due to the close relationship of this thesis to Sturm's thesis, the same concept of neighboring frames is utilized. As explained by Sturm, "Neighboring frames are just the frames that are easiest to transform into without first transforming into another defined frame" (11). Figure 3.4 presents the reference frames used in this thesis, where the arrows between frames represent two neighboring frames, and a transformation matrix that will be calculated in the next section. As Sturm points out, transforming to a non-neighboring frame is done by simply multiplying each transformation matrix that lies between the two frames together (11). In the following transformation matrices, sine(angle) and cosine(angle) are represented by s(angle) and c(angle), respectively. 


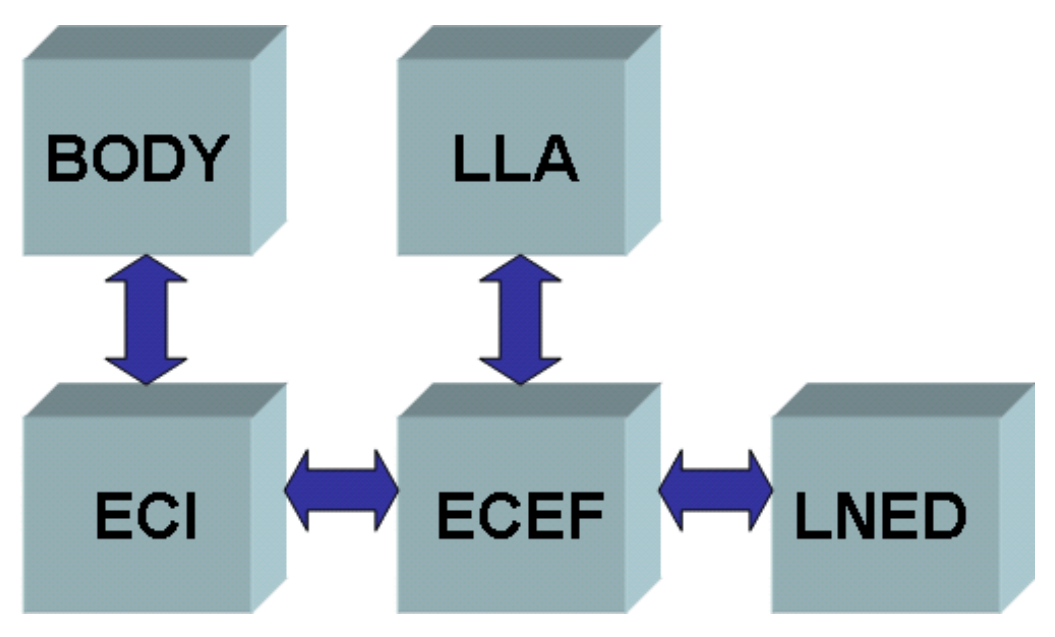

Figure 3.4. Neighboring Frames

\subsubsection{LNED to ECEF}

The transformation matrix to change a vector from the LNED frame to the ECEF frame requires latitude $(\varphi)$ and longitude $(\lambda)$ as displayed below.

$$
T_{L N E D \rightarrow E C E F}=\left[\begin{array}{ccc}
-s(\varphi) c(\lambda) & -s(\lambda) & -c(\varphi) c(\lambda) \\
-s(\varphi) s(\lambda) & c(\lambda) & -c(\varphi) s(\lambda) \\
c(\varphi) & 0 & -s(\varphi)
\end{array}\right]
$$

\subsubsection{LLA to ECEF}

Converting LLA information into a vector in the ECEF frame involves the semi-major axis (a) and the eccentricity (e) of the ellipsoid representation of the surface of the Earth, as shown in the following equations:

$$
\vec{x}_{E C E F}=\left[\begin{array}{c}
\left(\frac{a}{\kappa}+h\right) c(\varphi) c(\lambda) \\
\left(\frac{a}{\kappa}+h\right) c(\varphi) s(\lambda) \\
\left(\frac{a\left(1-e^{2}\right)}{\kappa}+h\right) s(\varphi)
\end{array}\right]
$$




$$
\kappa=\sqrt{1-e^{2} s^{2}(\varphi)}
$$

The altitude is being represented by ' $h$ ' since ' $a$ ' is being used for the semi-major axis.

\subsubsection{ECEF to ECI}

The conversion between the ECEF frame and the ECI frame is a single rotation about their z-axes using the angle between the First Line of Ares and the x-axis in the ECEF frame. To calculate this angle, one multiplies the angular rotation rate of the Earth $\left(\omega_{\oplus}\right)$ by the amount of time between the last Vernal Equinox and the time in question $(\Delta t)$. The transformation matrix is as follows:

$$
T_{E C E F \rightarrow E C I}=\left[\begin{array}{ccc}
c\left(w_{\oplus} \Delta t\right) & s\left(w_{\oplus} \Delta t\right) & 0 \\
-s\left(w_{\oplus} \Delta t\right) & c\left(w_{\oplus} \Delta t\right) & 0 \\
0 & 0 & 1
\end{array}\right]
$$

\subsubsection{ECI to Body}

One of the main goals of the PEKF is this conversion between the ECI frame and the spacecraft body frame. This transformation is characterized by a quaternion rotation, which only requires four pieces of information rather than the nine needed for a conventional rotation. A quaternion (q) can be contained in a 4 x 1 vector, whose information represents a unit vector and an angle for rotating about that unit vector $(\theta)$, as shown below:

$$
\bar{q}=\left[\begin{array}{c}
\vec{q} \\
q_{4}
\end{array}\right] \quad \text { where } \quad \vec{q}=\vec{p} \mathrm{~s}\left(\frac{\theta}{2}\right) \quad \text { and } \quad q_{4}=\mathrm{c}\left(\frac{\theta}{2}\right)
$$

The variable $\vec{p}$ represents the unit vector described above. The transformation used when dealing with quaternions is the following:

$$
A(\bar{q})=\left(q_{4}^{2}-\|\vec{q}\|^{2}\right) I_{3 x 3}+2 \vec{q} \vec{q}^{T}-2 q_{4}[\vec{q} \times]
$$




\subsection{Orbit Information}

\subsubsection{Satellite Tool Kit}

All of the orbit information for the satellite is acquired through AGI's Satellite Tool Kit (STK). Although Sturm created an orbit propagator, more work needs to be performed on it in order to get the accuracy required for this filter. STK uses a SGP4 orbit propagator to model the orbit of a satellite using Two Line Elements (TLEs) that are closest to the time periods desired. STK outputs latitudes, longitudes, altitudes, and sunlit time periods of the satellite.

\subsubsection{Universal Time Conversion}

Knowing the timestamp of downloaded data is crucial to the accuracy of the filter, since it determines the spacecraft's orbital position through the orbit propagator. The spacecraft's position creates the reference vectors that are compared to the actual data. CP3 contains an on-board clock that was set to Pacific Standard Time (PST). Unfortunately the clock was inaccurate and prone to drift, so it was difficult to determine the correct time stamp for data when it was downloaded. Three chunks of information are needed to approximate the PST of the data: the number of data points that the Command and Data Handling (C\&DH) could store, the exact PST that the data was downloaded, and the frequency of each data point. Once the PST of the data is discovered, it can easily be converted into Universal Time (UT) by subtracting the PST values by either 7 hours or 8 hours depending on the time of the year. UT is the time convention used in STK for orbit propagation. Higher accuracy of the orbit information is gained using the sunlit times provided by STK along with side panel data specifying 
similar information, such as solar panel currents. Aligning these two pieces of information will fine-tune the timestamps represented by the data.

\subsection{Reference Vector Definition}

\subsubsection{Magnetic Inertial Reference Vector}

As stated by Sturm, "The magnetic inertial reference vectors used in this thesis are calculated from the $10^{\text {th }}$ generation Inertial Geomagnetic Reference Field (IGRF-10) model" (17). The model is good from 2005 until 2010 with a decent accuracy during most of this time period. The model can become inaccurate if the measurements taken for it were disrupted by solar disturbances. To show that these disturbances did not occur for measured data used in the PEKF, the norms of the measured magnetic field vector and reference vector can be compared to check their alignment. In order to run this model, Maurice A. Tivey of the Woods Hole Oceanographic Institution created a Matlab m-file called magfd.m, which is used in this thesis. The m-file uses longitude, altitude, time, and co-latitude $\left(90^{\circ}-\right.$ latitude) as inputs. It outputs a $4 \times 1$ vector containing the magnetic field vector in the LNED frame and the magnitude of the magnetic field. The magnetic field vector is converted into the ECI frame, making it the Magnetic Inertial Reference Vector (MIRV), using the neighboring frame transformations.

\subsubsection{Solar Inertial Reference Vector}

The solar inertial reference vector (SIRV) is a unit vector pointing from the Sun to the Earth in inertial space. Since the distance from the satellites in orbit to the center of the Earth is orders of magnitude smaller than the distance from the Earth to the Sun, there will be a very minimal loss in accuracy in having this vector point to the Earth rather than the satellite. The SIRV is calculated using equations from the 2000 Astronomical 
Almanac, which gives the position of the sun in the sky to an accuracy of 0.01 degrees between 1950 and 2050 (C1-C2, C24). The equations use an elliptical Earth orbit that takes the change of obliquity of the ecliptic with time into account. First, the Mean anomaly (g) and the Mean Longitude (W) of the Sun are calculated:

$$
\begin{gathered}
g=357.528+0.9856003 d \\
W=280.461+0.9856474 d
\end{gathered}
$$

where the variable $\mathrm{d}$ refers to the number of days before J2000. If the Mean Longitude or Mean anomaly of the Sun are not between 0 and 360 degrees, 360 degrees is added or subtracted from the calculated value until they fall within that range. Next, the ecliptic longitude of the Sun $(\Lambda)$ and the obliquity of the ecliptic plane $(\beta)$ are determined:

$$
\begin{gathered}
\Lambda=W+1.915 \sin (g)+0.02 \sin (2 g) \\
\beta=23.439-0.0000004 d
\end{gathered}
$$

These angles are used to find the Right Ascension $(\mu)$ and Declination $(\delta)$ of the Sun, as illustrated below:

$$
\begin{gathered}
\mu=\tan ^{-1}\left(\frac{Y}{X}\right) \\
Y=\cos (\beta) \sin (\Lambda) \\
X=\cos (\Lambda) \\
\delta=\sin ^{-1}(\sin (\beta) \sin (\Lambda))
\end{gathered}
$$

The Right Ascension has some conditions based on the $\mathrm{X}$ and $\mathrm{Y}$ variables displayed above:

$$
\text { If } X<0 \text { then } \mu=\mu+180
$$

If $Y<0$ and $X>0$ then $\mu=\mu+360$ 
The Right Ascension and Declination of the Sun are converted into the SIRV through the following equation:

$$
S I R V=-\left[\begin{array}{l}
\mathrm{c}(\alpha) \\
\mathrm{s}(\alpha) \\
\mathrm{s}(\delta)
\end{array}\right]
$$

Once created, the SIRV is utilized as a verification method for the filter by using the output quaternions to rotate it into the spacecraft body frame, becoming the solar body estimated vector (SBEV). The SBEV is passed through the thermal model, which will be compared with actual side panel temperature data. The SBEV is also used to estimate the solar panel power using its angle to each of the side panels, which is compared with actual solar panel current data.

\subsubsection{Cubesat Inertial Reference Vector}

The cubsat inertial reference vector (CIRV) refers to the unit vector from the center of the Earth to the cubesat in inertial space. It is created using the latitude, longitude, and altitude information from STK and the frame transformations from LLA to ECI. Much like the SIRV, the CIRV is passed into the filter where it is rotated into the spacecraft body frame using the output quaternions. The CIRV becomes the cubsat body estimated vector $(\mathrm{CBEV})$ and becomes an input into the thermal model for albedo and Earth infrared (IR) effects.

\subsection{Sensor Calibration}

The PEKF utilizes two types of sensors onboard the spacecraft, magnetometers and thermistors. Honeywell HMC 1052 two-axis magnetometers were employed due to their heritage on previous cubesats. The thermistor is a resistor soldered to both sides of a side panel whose resistance changes with temperature. There is one 2-axis magnetometer and 
two thermistors on five of the six sides of the cubesat. The payload face does not contain any sensors. The backside of a side panel is presented in Figure 3.5, with the inside twoaxis magnetometer and thermistor highlighted.

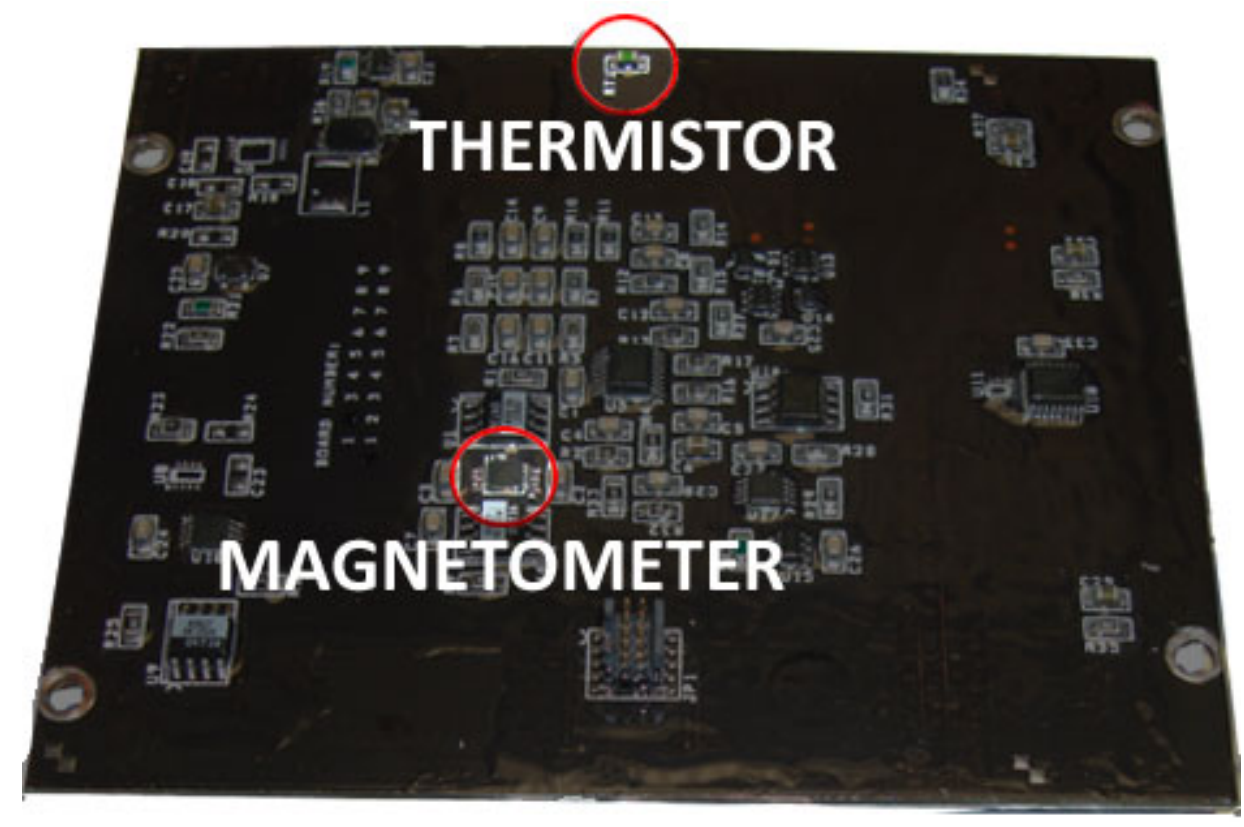

Figure 3.5. CP3 Side Panel Sensors

\subsubsection{Magnetometers}

The same magnetometer calibration as described in Sturm's thesis was utilized on each of the side panels. The magnetometers output voltage readings that are converted into 8-bit readings from $0-255$. The calibration ensures that these 8 -bit readings are within the range of the maximum magnetic field present in orbit and that 128 bits corresponds to a reading of zero Gauss. To calibrate the magnetometers, each side panel is placed on an acrylic board with lines that indicate the axis of the magnetometer. The acrylic board is placed on a compass that is set up with $0^{\circ}$ facing true North. Readings are then taken from the magnetometer at each of the different angles indicated on the compass, as well 
as from a FVM-400 vector magnetometer. This process is demonstrated in Figure 3.6. from Sturm's thesis (23).

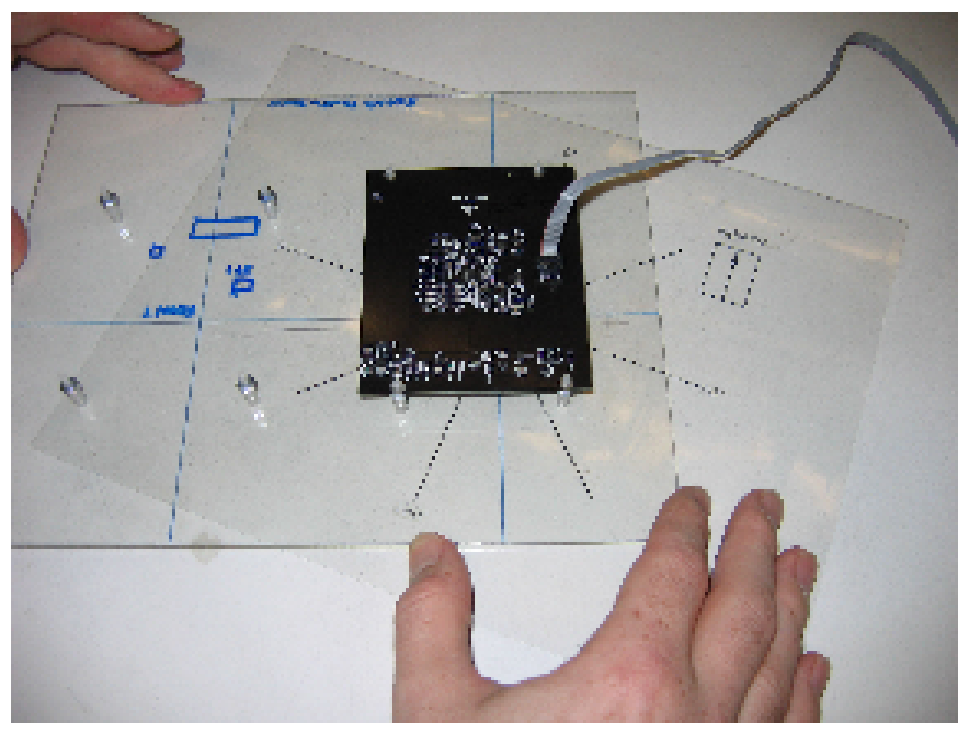

Figure 3.6. Side Panel Magnetometer Calibration

Each magnetometer has three resistor values that could be changed to calibrate them.

Two of those resistors control the gains for the two magnetometers, and the third resistor controls the zero value for both magnetometers together. Sturm created a Microsoft Excel tool that assists with the calibration by indicating the values for resistor changes, as demonstrated in Figure 3.7. As Sturm points out, "The circle is sized such that the difference in magnetic field strength on the ground and in orbit is taken into account; therefore, a value lying on that circle will not result in saturated values during in-orbit operation" (24). The "X" pattern created by the sets of parallel lines indicates the values that the zero reading will take if that resistor is changed, with the desired value falling within the diamond in the center. If the zero reading does not fall within that " $\mathrm{X}$ " pattern, then that magnetometer cannot be used. Once the proper resistor value is in place that places the zero reading within the diamond, the rest of the readings are taken and should 

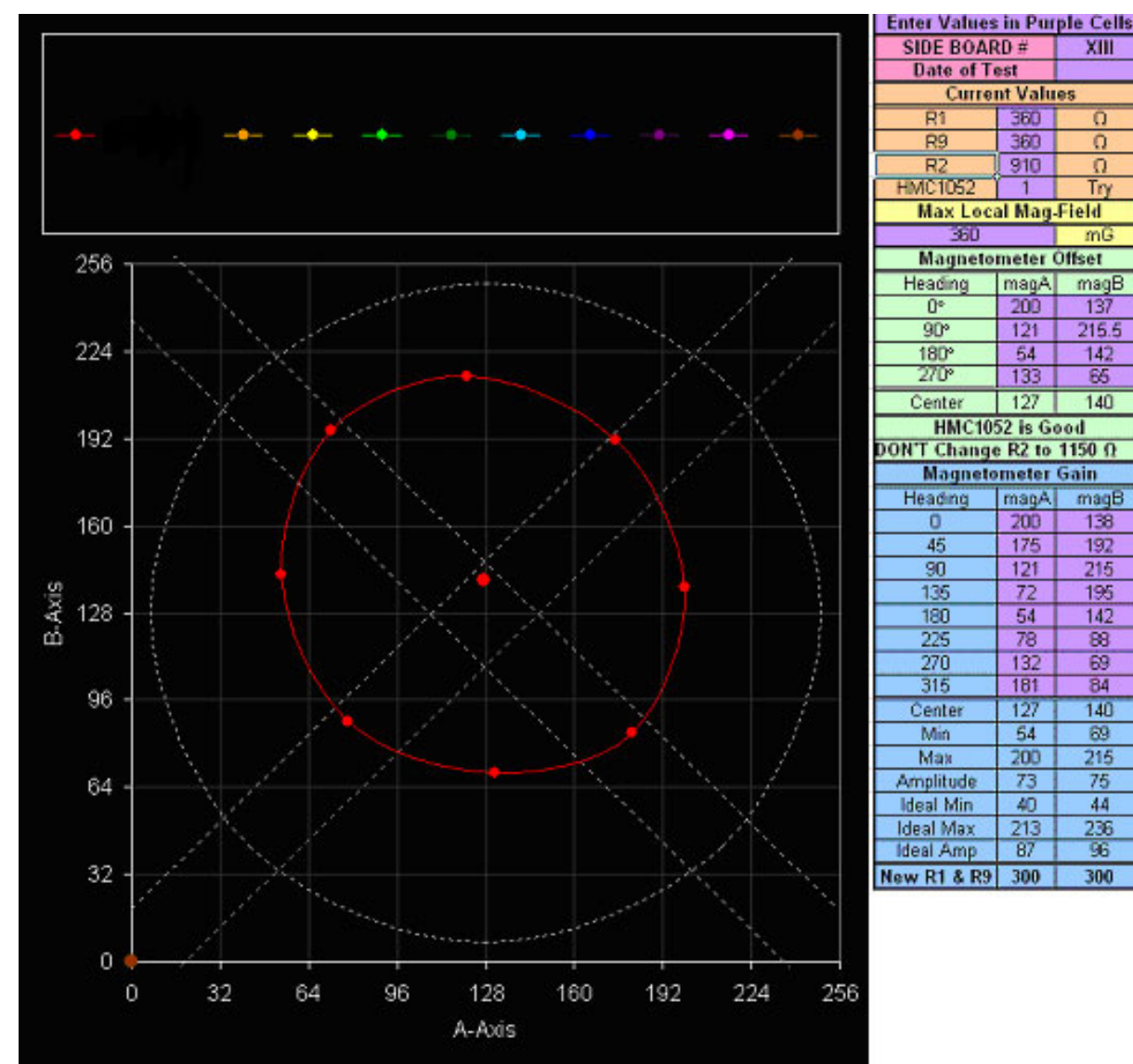

Magnetometer Offset \begin{tabular}{|c|c|c|}
\hline Heading & magA \\
\hline $0^{\circ}$ & magB \\
\hline
\end{tabular} \begin{tabular}{|c|c|c|}
\hline $0^{\circ}$ & 200 & 137 \\
\hline $90^{\circ}$ & 121 & 215.5 \\
\hline
\end{tabular} \begin{tabular}{|c|c|c|}
\hline $180^{\circ}$ & 54 & 142 \\
\hline $270^{\circ}$ & 133 & 85 \\
\hline
\end{tabular} \begin{tabular}{c|c|c}
\hline Center & 127 & 140 \\
\hline HMC 1052 is 6004
\end{tabular} HMC 1052 is Good
DONT Change R2 to 1150 n? Maqnetometer Gain \begin{tabular}{|c|c|c|}
\hline Heading & magA & mag \\
\hline 0 & 200 & 138 \\
\hline 45 & 175 & 192 \\
\hline
\end{tabular} \begin{tabular}{|l|l|l|}
\hline 0 & 200 & 138 \\
\hline 45 & 175 & 192 \\
\hline 90 & 121 & 215 \\
\hline
\end{tabular} \begin{tabular}{|c|c|c|}
\hline 90 & 121 & 215 \\
\hline 135 & 72 & 195 \\
\hline
\end{tabular} \begin{tabular}{|l|l|l|}
\hline 180 & 54 & 142 \\
\hline
\end{tabular} \begin{tabular}{|c|c|c|}
\hline 225 & 78 & 88 \\
\hline 270 & 132 & 69 \\
\hline
\end{tabular} \begin{tabular}{|c|c|c|}
\hline 315 & 181 & 84 \\
\hline Center & 127 & 140 \\
\hline
\end{tabular} \begin{tabular}{|c|c|c|}
\hline Center & 127 & 140 \\
\hline Min & 54 & 60 \\
\hline
\end{tabular} \begin{tabular}{|c|c|c|}
\hline Min & 54 & 69 \\
\hline Mas & 200 & 215 \\
\hline
\end{tabular} \begin{tabular}{|l|c|c|}
\hline Amplitude & 73 & 75 \\
\hline
\end{tabular} \begin{tabular}{|l|r|r}
\hline Ideal Min & 40 & 44 \\
\hline
\end{tabular} \begin{tabular}{|c|c|c|}
\hline Ideal Max & 213 & 236 \\
\hline Ideal Amp & 87 & 96 \\
\hline
\end{tabular} \begin{tabular}{|l|c|c|}
\hline New R1 \& R9 & 300 & 300 \\
\hline
\end{tabular}

Figure 3.7. Magnetometer Calibration Excel Tool

lie on the circle. If they do not, the appropriate resistor values should be changed according to the Excel tool. All ten of the magnetometer readings together are used to form the estimated magnetic field vector in the body frame, $\hat{\vec{b}}^{\text {Body }}$. The scalar magnetometer readings for each axis are averaged together and placed under the appropriate axis in the estimated magnetic field vector.

\subsubsection{Thermistors}

Pre-flight testing of the thermistors on CP3 involved a simple test of whether it would read a temperature change in the appropriate direction when it was heated up or cooled down. A more involved characterization was instituted after the launch of CP3, which 
involves taking temperature readings while the satellite is undergoing thermal cycling in a thermal vacuum chamber. A thermal vacuum characterization of the same thermistors was completed on a next generation cubesat, which showed the thermistors to be fairly accurate to within a degree Celsius.

\subsection{CP3 Downloadable Data}

\subsubsection{Programmed Data Packages}

CP3 allocates a certain amount of memory for two types of downloadable data packages. These data packages run on a circular buffer, which has the new data snapshots replacing the oldest ones that are stored. The default snapshot rates are set at two minutes, but they can be changed using uplink commands to the fastest rate of ten seconds. The first type of data package is designated C\&DH data, which includes data from every sensor onboard the spacecraft except the payload. CP3 can hold 650 sensor snaps of C\&DH data. The second type of data package is called ADC data, which is a shortened version of the $\mathrm{C} \& \mathrm{DH}$ data with only limited satellite health data. By restricting the data to attitude determination sensors and some health data, CP3's memory can hold 2880 sensors snaps of ADC data. The data from both packages that is the most valuable to this thesis comes from the magnetometers, external thermistors, and solar panel current sensors.

\subsubsection{Mitigating Dropped Packet Effects}

During a data download from CP3, low signal strength or noise can disrupt the signal from reaching the ground station. This leads to dropped packets in the data, which can lead to divergence in the PEKF. In order to fill in the dropped packets, the fit.m Matlab function was utilized. Each set of data downloaded from one pass of the satellite is fit to 
an $8^{\text {th }}$ degree Fourier series, and the dropped packets were filled in with the resulting function. The process does not perfectly fill in the data, but it is enough to keep the PEKF from diverging due to dropped packets.

\subsection{PEKF Equations}

\subsubsection{Equations of Motion}

The equations of motion of a spacecraft are applied to the PEKF in order to propagate the states from one time step to the next after each filter run. This propagation is accomplished using a numerical Runge-Kutta integrator in Matlab. The equations of motion stem from Euler's equation for the specific angular momentum of a spacecraft (L), as presented below:

$$
\dot{\vec{L}}=-\vec{\omega} \times \vec{L}
$$

Through some assumptions and manipulation, this equation can be simplified in order to reduce computational time. As demonstrated below, Euler's equation can be rearranged in terms of the time derivative of the spacecraft's angular velocity $(\omega)$ using the relationship between specific angular momentum and the moments of inertia matrix $(\mathrm{J})$.

$$
\dot{\vec{\omega}}=-J^{-1}(\vec{\omega} \times J \vec{\omega})
$$

This can be further simplified by assuming that the spacecraft's body axes are the principle axes for the moments of inertia, which is illustrated in the following equations.

$$
\begin{aligned}
& \dot{\omega}_{1}=\frac{\left(J_{2}-J_{3}\right)}{J_{1}} \omega_{2} \omega_{3} \\
& \dot{\omega}_{2}=\frac{\left(J_{3}-J_{1}\right)}{J_{2}} \omega_{3} \omega_{1} \\
& \dot{\omega}_{3}=\frac{\left(J_{1}-J_{2}\right)}{J_{3}} \omega_{1} \omega_{2}
\end{aligned}
$$


The equations that represent the quaternion time derivative with respect to the angular velocity are:

$$
\begin{gathered}
\dot{q}=\frac{1}{2} \Omega(\vec{\omega}) q \\
\Omega(\vec{\omega})=\left[\begin{array}{cccc}
0 & \omega_{3} & -\omega_{2} & \omega_{1} \\
-\omega_{3} & 0 & \omega_{1} & \omega_{2} \\
\omega_{2} & -\omega_{1} & 0 & \omega_{3} \\
-\omega_{1} & -\omega_{2} & -\omega_{3} & 0
\end{array}\right]
\end{gathered}
$$

The $\Omega$ matrix represents a quaternion multiplication without a $\mathrm{q}_{4}$ term.

\subsubsection{Reduced State and Measurement Equations}

The full state vector for the PEKF is:

$$
\vec{x}=\left[\begin{array}{l}
q \\
\vec{\omega}
\end{array}\right]
$$

As Erick points out, a reduced body-fixed state vector can be used in order to alleviate problems with the covariance matrix $\mathrm{P}$ and quaternion normalization issues due to the redundancy of the four quaternion variables for the rotation (42). The following reduced state vector replaces the quaternion with $\delta \vec{q}$, "the vector component of the error quaternion that transforms the estimated full quaternion to the actual quaternion" (Sturm $42)$.

$$
\begin{gathered}
\tilde{x}=\left[\begin{array}{c}
\delta \vec{q} \\
\vec{\omega}
\end{array}\right] \\
\bar{q}=\delta \vec{q} \otimes \hat{\bar{q}}
\end{gathered}
$$


The $\otimes$ symbol represents a quaternion multiplication, and $\hat{\bar{q}}$ is the full quaternion estimate. Since $\delta \vec{q}$ is an error quaternion vector, its magnitude should be less than one and approach zero as the filter converges, allowing the $\delta q_{4}$ term to be calculated as follows:

$$
\delta q_{4}=\sqrt{1-\|\delta \vec{q}\|^{2}}
$$

When the filter begins running, the magnitude of the error quaternion vector could be greater than one, which would create an imaginary $\delta \mathrm{q}_{4}$ term. Humphrey of Utah State University developed a formula for the whole error quaternion to combat this situation, which is stated below:

$$
\delta q=\frac{1}{\sqrt{1+\|\delta \vec{q}\|^{2}}}\left[\begin{array}{c}
\delta \vec{q} \\
1
\end{array}\right]
$$

Humphrey derives the following relationship between the error quaternion and the regular quaternion, which gives rise to the reduced state dynamic equations:

$$
\begin{aligned}
& {\left[\begin{array}{c}
\delta \vec{q} \\
\vec{\omega}
\end{array}\right]=\left[\begin{array}{cc}
\Xi^{T}(\hat{q}) & 0_{3 \times 3} \\
0_{3 \times 4} & I_{3 \times 3}
\end{array}\right]\left[\begin{array}{c}
q \\
\vec{\omega}
\end{array}\right]} \\
& \Xi(\hat{q})=\left[\begin{array}{ccc}
q_{4} & -q_{3} & q_{2} \\
q_{3} & q_{4} & -q_{1} \\
-q_{2} & q_{1} & q_{4} \\
-q_{1} & -q_{2} & -q_{3}
\end{array}\right]
\end{aligned}
$$

The reduced state dynamic equations are:

$$
\delta \dot{\vec{q}}=\frac{1}{2} \Omega(\vec{\omega}) \delta \vec{q}-\frac{1}{2} \delta \vec{q} \otimes \hat{\bar{\omega}} \text { where } \bar{\omega}=\left[\begin{array}{c}
\vec{\omega} \\
0
\end{array}\right] \text { (Humphrey 11) }
$$

The relationship between the body and inertial magnetic field vectors is a rotation using the estimated quaternion and the error quaternion vectors, as demonstrated by: 


$$
\vec{b}^{\text {Body }}=A(\delta \bar{q}) A(\hat{\bar{q}}) \vec{b}^{\text {Interial }}
$$

Using the reduced quaternion state and the relationship described above, Sturm derives the following measurement equation using the estimated magnetic field vector in the body frame:

$$
h(\tilde{x})=I_{3 \times 3}+2\left[\hat{\vec{b}}^{B o d y} \times\right] \delta \vec{q}
$$

The expression in brackets in the previous equation refers to the cross product matrix, which can be applied to any vector. The cross product matrix is displayed below using $\hat{\vec{b}}^{B o d y}$ as the vector.

$$
\left[\hat{\vec{b}}^{\text {Body }} \times\right]=\left[\begin{array}{ccc}
0 & -\hat{b}_{3}^{\text {Body }} & \hat{b}_{2}^{\text {Body }} \\
\hat{b}_{3}^{\text {Body }} & 0 & -\hat{b}_{1}^{\text {Body }} \\
-\hat{b}_{2}^{\text {Body }} & \hat{b}_{1}^{\text {Body }} & 0
\end{array}\right]
$$

\subsubsection{Matrix Derivations}

Once the model and states are defined, the various matrices that appear in the EKF equations in Chapter 2 can be calculated. The linear system dynamics matrix $\left(\mathrm{F}_{\mathrm{i}}\right)$ and the measurement matrix $\left(\mathrm{H}_{\mathrm{i}}\right)$ were the two matrices that required a linearization of the state and measurement equations, as shown below:

$$
F_{i}=\left[\begin{array}{cc}
-[\hat{\omega} \times] & \frac{1}{2} I_{3 \times 3} \\
0 & \Theta(\hat{\omega})
\end{array}\right] \text { where } \Theta(\hat{\omega})=\left[\begin{array}{ccc}
0 & \frac{\hat{\omega}_{3}\left(J_{2}-J_{3}\right)}{J_{1}} & \frac{\hat{\omega}_{2}\left(J_{2}-J_{3}\right)}{J_{1}} \\
\frac{\hat{\omega}_{3}\left(J_{3}-J_{1}\right)}{J_{2}} & 0 & \frac{\hat{\omega}_{1}\left(J_{3}-J_{1}\right)}{J_{2}} \\
\frac{\hat{\omega}_{2}\left(J_{1}-J_{2}\right)}{J_{3}} & \frac{\hat{\omega}_{1}\left(J_{1}-J_{2}\right)}{J_{3}} & 0
\end{array}\right]
$$




$$
H_{i}=\left[2\left[\begin{array}{ll}
\left.\hat{\vec{b}}_{i}^{\text {Body }} \times\right] & 0_{3 \times 3}
\end{array}\right]\right.
$$

The linear system dynamics matrix is used to approximate the state transition matrix $(\Phi)$ during each filter loop. The measurement noise matrix (R) depends on the squared standard deviation of the averaged magnetometer readings, as shown below:

$$
R=\sigma_{\text {AvgMag }}^{2} I_{3 \times 3}
$$

\subsubsection{Filter Initialization}

Initial states and matrix values are needed to start the PEKF. Choosing these initial values is critical in ensuring filter convergence. Sturm derives the following initial conditions for the states:

$$
\begin{gathered}
\hat{\bar{q}}_{0}^{T}=\left[\begin{array}{llll}
0 & 0 & 0 & 1
\end{array}\right] \\
\hat{\vec{\omega}}_{0}=\frac{\vec{b}_{1}^{\text {Body }} \times \frac{\vec{b}_{2}^{\text {Body }}-\vec{b}_{1}^{\text {Body }}}{T_{s}}}{\left\|\vec{b}_{1}^{\text {Body }}\right\|^{2}}
\end{gathered}
$$

The initial quaternion estimates the alignment of the body frame and the ECI frame. The initial body rate estimate is approximated by the difference of two consecutive body magnetic field vector measurements divided by the frequency of those measurements. The initial process noise matrix $(\mathrm{Q})$ and covariance matrix $(\mathrm{P})$ are defined below:

$$
\begin{aligned}
& P_{0}=P I_{6 \times 6} \\
& Q_{0}=Q I_{6 \times 6}
\end{aligned}
$$

Both matrices depend on a scalar value that is chosen to initialize the PEKF. Choosing these scalars in order to ensure filter convergence is known as tuning the filter. 


\section{CHAPTER 4: SATELLITE THERMAL MODEL}

\subsection{On-orbit Temperature Trends}

During the first few months after launch, CP3 had external temperatures ranging from $-30^{\circ} \mathrm{C}$ to $20^{\circ} \mathrm{C}$, as shown in Figure 4.1. Six months after launch, the external

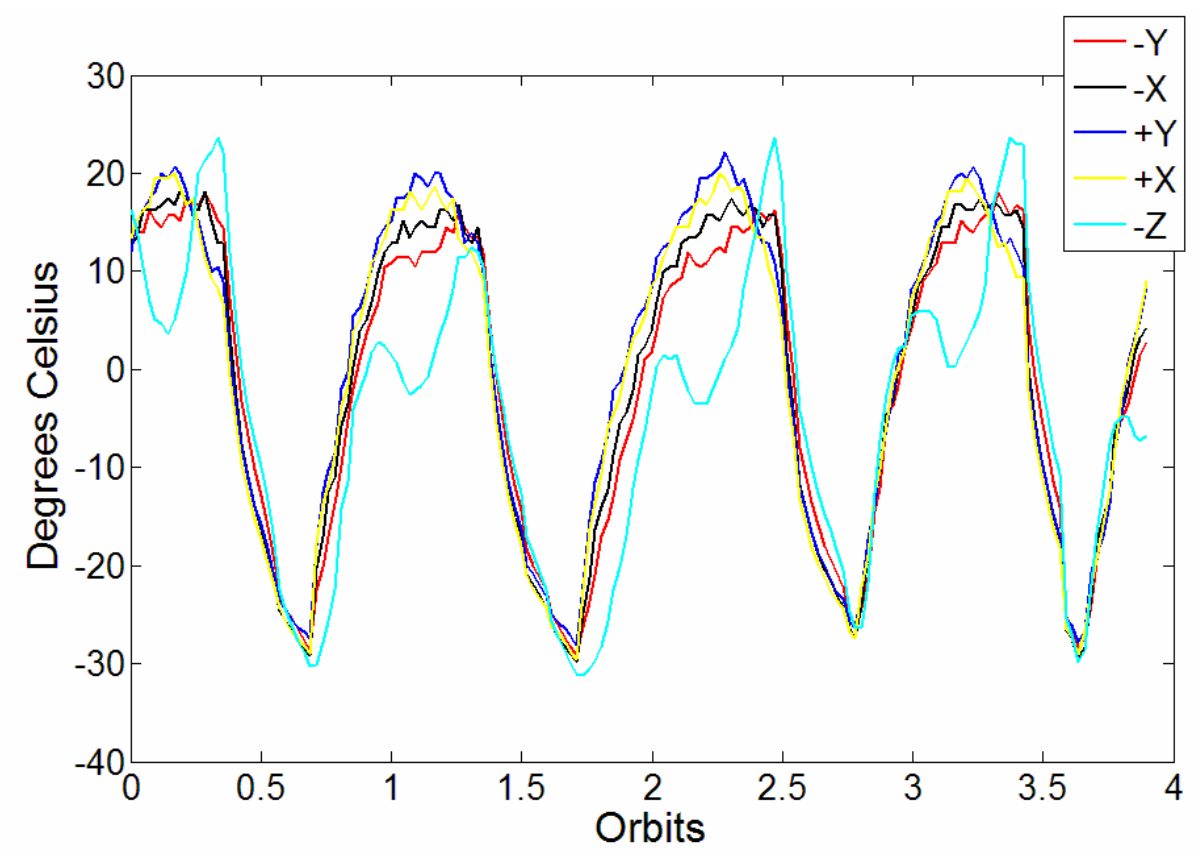

Figure 4.1. CP3 Side Panel Temperature Data for June $18^{\text {th }}, 2007$

temperature range expanded to between $-30^{\circ} \mathrm{C}$ and $50^{\circ} \mathrm{C}$, which is demonstrated by

Figure 4.2. This led to the conjecture that the satellite's rotation rate was slowing down and that one could use the temperature data as attitude information. With the proper thermal model, the side panel temperature data could work as a crude sun sensor. 


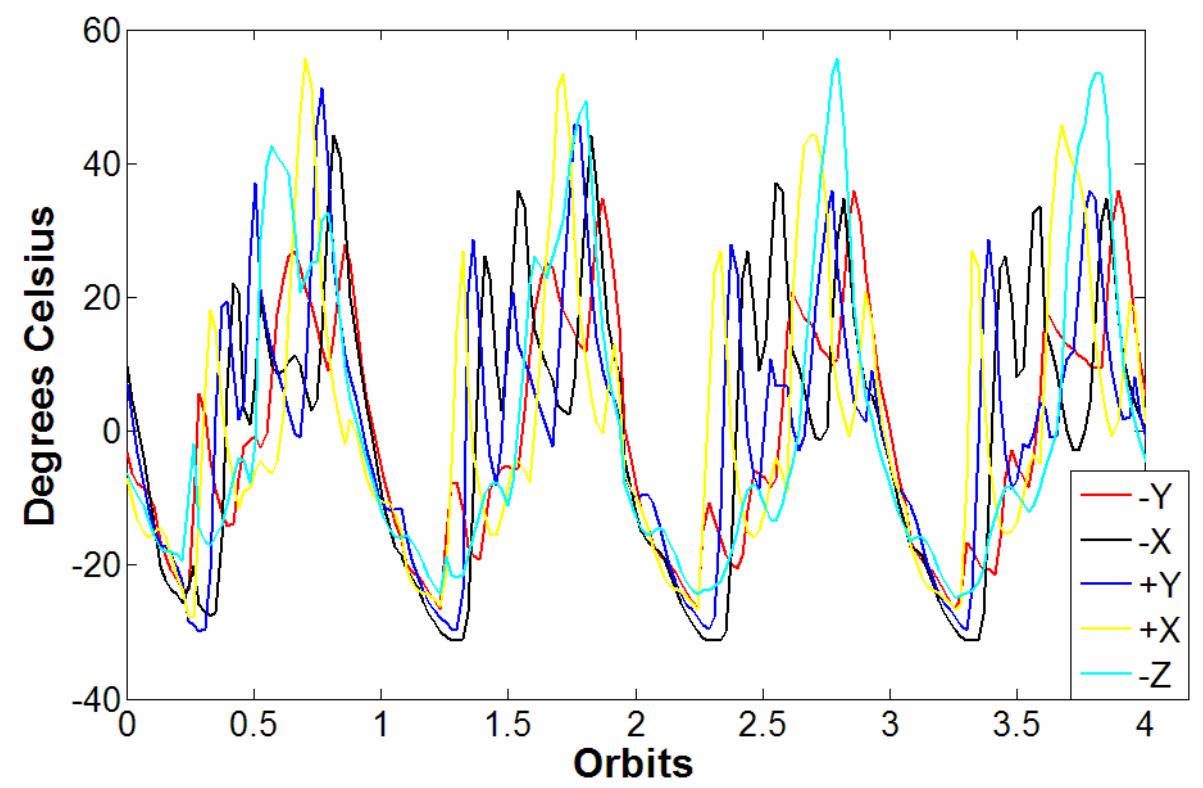

Figure 4.2. CP3 Side Panel Temperature Data for November $18^{\text {th }}, 2007$

\subsection{Model Assumptions}

\subsubsection{Node Definitions}

The nodes for the thermal model were built around the available information from onorbit data. Since there are only five side panels out of the six that give temperature data, the thermal model has five external nodes and one internal node. Each external node has the same dimensions as a CP3 side panel and is composed of printed circuit board (PCB) material, stainless steel, and solar cells. The internal node is modeled as a solid cube with a composition of aluminum, PCB material, batteries, and thermal mass. The thermal mass was added to the internal node to account for the inaccuracies of modeling the internal workings of the cubesat as a solid cube.

In order to develop a thermal model, thermal constants for the materials in the nodes had to be acquired. These values are considered common knowledge or they appear in many 
sources for some of the materials used. The rest of the thermal constants came from specific sources or they were assumed based on similar materials, as signified in Table 4.1, which contains all of the thermal constants used for the thermal model.

Table 4.1. Thermal Model Constants

\begin{tabular}{|c|c|c|c|c|c|}
\hline & $\begin{array}{c}\text { Absorbtivity, } \\
\alpha\end{array}$ & $\begin{array}{c}\text { Emissivity, } \\
\varepsilon\end{array}$ & $\begin{array}{c}\text { Thermal } \\
\text { Conductivity, } \\
\mathrm{k}\end{array}$ & $\begin{array}{l}\text { Specific Heat } \\
\text { Capacity, } \\
\text { c }(\mathrm{J} / \mathrm{kg}-\mathrm{K})\end{array}$ & $\begin{array}{l}\text { Density, } \\
\rho\left(\mathrm{kg} / \mathrm{m}^{3}\right)\end{array}$ \\
\hline $\begin{array}{l}\text { Stainless } \\
\text { Steel }\end{array}$ & 0.15 & 0.4 & 16.5 & 500 & 8000 \\
\hline Solar Cells & $\begin{array}{c}0.92 \\
(\text { Spectrolab) }\end{array}$ & $\begin{array}{c}0.85 \\
\text { (Spectrolab) }\end{array}$ & $\mathrm{N} / \mathrm{A}$ & 0.01 & $\begin{array}{c}6000 \\
(\text { Spectrolab) }\end{array}$ \\
\hline PCB & 0.25 & 0.8 & $\begin{array}{c}0.268 \\
\text { (Shaybany 2) }\end{array}$ & 1136 & 2145 \\
\hline $\begin{array}{c}\text { Thermal } \\
\text { Mass }\end{array}$ & 0.25 & 0.8 & N/A & 1136 & 2145 \\
\hline $\begin{array}{l}\text { Aluminum } \\
\text { 7075-T6 }\end{array}$ & $0.3(\operatorname{Le} 2)$ & $0.2(\operatorname{Le~} 2)$ & N/A & 9600 & 2810 \\
\hline $\begin{array}{l}\text { Lithium Ion } \\
\text { Battery } \\
\text { (Chen 116) }\end{array}$ & 0.1 & 0.25 & N/A & 1000 & 2528 \\
\hline
\end{tabular}

As indicated in Table 4.1, the thermal mass used in the internal node was assumed to have similar thermal constants as the PCB material, since that is what makes up a majority of the internal mass of the satellite. Another thing to notice is the two thermal conductivity constants, which stem from the assumption that the conduction only occurs through the side panel PCB and stainless steel bolts. When referring to the table, a reference called out in an individual cell explains the origin of that value. A reference displayed with the name of a material denotes that all the values in that row come from that reference. Any cell or row without a reference was either assumed or considered common knowledge.

Each material has two composition factors associated with it: one based on volume $\left(\mathrm{F}_{\mathrm{V}}\right)$ and one based on surface area $\left(\mathrm{F}_{\mathrm{A}}\right)$. They are the decimal percentage of the material in the volume or on the surface of the node. The composition factors based on volume 
correspond to delegating constants for density and specific heat in the governing equation. The surface area based composition factors delegate constants in the radiation heat transfer equations.

\subsubsection{Node Heat Transfer Connections}

The thermal model is setup with two modes of heat transfer: radiation and conduction. Solar radiation, albedo and Earth's infrared radiation (IR), and radiation from all of the other nodes affect every node. The internal node is connected to all of the external nodes through conduction. Figure 4.3 demonstrates the node connections using the internal node and two external nodes for simplicity. The solar radiation, Earth's IR, and albedo affect the internal node consistently as long as

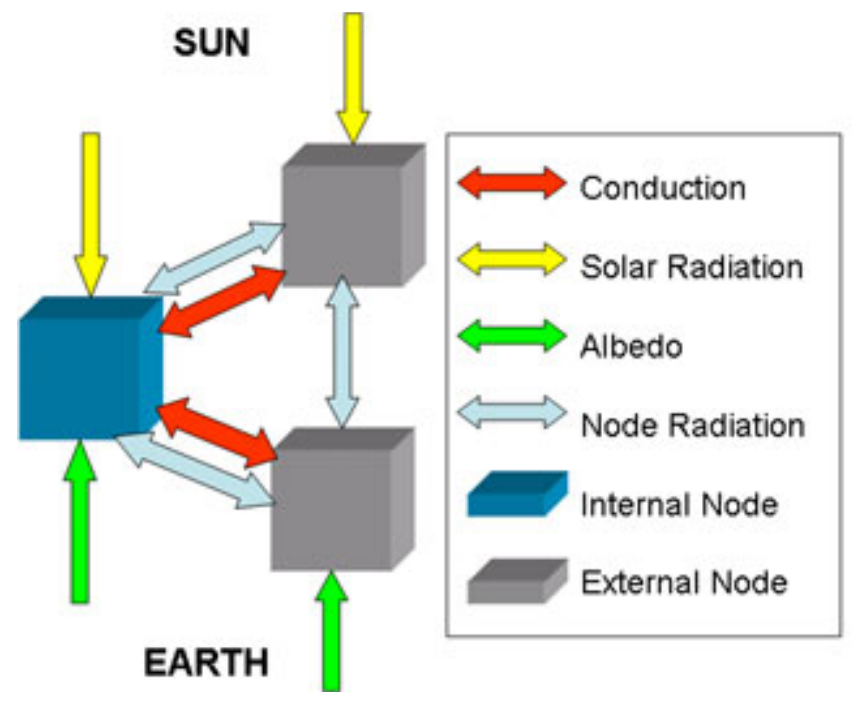

Figure 4.3 Thermal Node Connections

the satellite is in the sun. For the external nodes, the solar radiation, Earth's IR, and albedo effects depend on the angle of the SIRV and CIRV to each node.

\subsubsection{Equation Derivation}

The fundamental equation used to relate the nodes defined in the previous section is the first law of thermodynamics. By assuming that each node is contained in a closed system 
and that it is an incompressible solid with a fixed mass, the following form of the first law of thermodynamics can be utilized:

$$
\rho V c \frac{d T}{d t}=\dot{Q}+\dot{Q}_{V}
$$

This form of the equation represents the rate temperature changes with time $\left(\frac{d T}{d t}\right)$ with respect to volume $(\mathrm{V})$, density $(\rho)$, specific heat $(\mathrm{c})$, heat transfer into the system $(\dot{Q})$, and heat generated from within the system $\left(\dot{Q}_{V}\right)$. The next step in building the thermal model was defining the terms in the governing equation. The volume, specific heat, and density for each node were based on the thermal constants in Table 4.1. An assumption was made that there is no heat generated within each system, even though the batteries in the internal node produce some heat. This assumption was made to account for the internal node's simplified representation. Since the internal node is modeled as a solid cube, any heat generated there would have substantial affects on its temperature. The only variable left to define is the heat transfer into the system. It can be broken up into the five types of heat transfer: solar radiation $\left(\dot{Q}_{S R}\right)$, albedo and Earth's IR, emitted radiation $\left(\dot{Q}_{E R}\right)$, thermal conduction $\left(\dot{Q}_{C}\right)$, and radiation absorbed by other nodes $\left(\dot{Q}_{A R}\right)$. These assumptions along with the composition factors for the materials update the governing equation to the following:

$$
\frac{d T}{d t}=\frac{\dot{Q}_{S R}+\dot{Q}_{A}+\dot{Q}_{C}+\dot{Q}_{E R}+\dot{Q}_{A R}}{V\left(F_{V 1} \rho_{1} c_{1}+\ldots F_{V n} \rho_{n} c_{n}\right)}
$$

The variable $\mathrm{n}$ represents the number of materials in the node, which is either 3 for the external nodes or 4 for the internal node. 


\subsubsection{Solar Radiation}

Electromagnetic waves being emitted from one body and absorbed by another causes radiation. The equation below models heat transfer by radiation:

$$
\dot{Q}_{R}=\alpha A \sigma T^{4} \text { or } \dot{Q}_{R}=\varepsilon A \sigma T^{4}
$$

where A, $\alpha$, and $\varepsilon$ are the surface area, absorbtivity, and emissivity of the system, respectively. The Stefan-Boltzmann constant, $\sigma$, comes from the Stefan-Boltzmann Law:

$$
G=E_{b}=\sigma T^{4}
$$

where $E_{b}$ represents the total emissive power of a black body giving off radiation and $G$ is the irradiation on the surface of a body. In order to model solar radiation, the solar constant of 1353 watts per squared meter is used for the Sun, which is denoted by the variable $\mathrm{G}_{\mathrm{O}}$. This gives rise to the following equation used to represent solar radiation for the external nodes on CP3:

$$
\dot{Q}_{S R}=\left(F_{A 1} \alpha_{1}+\ldots F_{A n} \alpha_{n}\right) A G_{O} \cos (\eta)
$$

The angle $\eta$ represents the incidence angle of the sun vector on each side panel. The internal node uses the same equation without the cosine term. All of the nodes conditionally calculate this value when the satellite is in the Sun. The external nodes only calculate it if the Sun vector is between $0^{\circ}$ and $90^{\circ}$ to the normal vector of the side panel. One assumption to note is that the front panel with the antenna mount takes a $25 \%$ reduction in heat transfer from solar radiation since the antenna mount blocks the solar cells from getting sun at low incidence angles.

\subsubsection{Albedo and Earth's Infrared Radiation}

Albedo is the irradiation of solar radiation off of the surface of the Earth due to the Earth's reflectivity. The Earth also emits radiation in the form of infrared, which also 
produces heat. They were combined for this mode of heat transfer for simplicity. They use the same equation as solar radiation with one exception. The solar constant is scaled down by two factors: $30 \%$ when the satellite is in the sun and $10 \%$ when it is not in the sun. The nodes experience the radiation in the same manner as solar radiation. The front panel node also takes the same $25 \%$ hit to its radiation heat transfer.

\subsubsection{Emitted Radiation}

The emitted radiation is the only form of heat transfer that removes heat from the system. It is characterized by the following equation:

$$
\dot{Q}_{E R}=\left(F_{A 1} \varepsilon_{1}+\ldots F_{A n} \varepsilon_{n}\right) A \sigma T^{4}
$$

All of the nodes emit radiation at all times, contributing to the cyclic nature of the thermal model.

\subsubsection{Thermal Conductance}

All of the external nodes are connected to the internal node through conduction. The conduction occurs between the outside and inside of the side panels, assuming that the temperature of the internal node is always measured at its outer edges. The internal node conduction is defined by:

$$
\dot{Q}_{C}=\frac{A\left(F_{V 1} k_{1}+\ldots F_{V n} k_{n}\right)}{l}\left(\left(T_{e x t 1}-T_{\text {int }}\right)+\ldots\left(T_{e x t 5}-T_{\text {int }}\right)\right)
$$

The variable $\mathrm{k}$ is known as the thermal conductivity of the materials in the node and 1 is the thickness of the side panel. The external nodes use the following equation:

$$
\dot{Q}_{C}=\frac{A\left(F_{V 1} k_{1}+\ldots F_{V n} k_{n}\right)}{l}\left(T_{\text {int }}-T_{e x t}\right)
$$




\subsubsection{Absorbed Radiation from Other Nodes}

In order to evaluate the absorbed radiation from all of the nodes, the shape factor for all of the nodes has to be calculated. A shape factor is the fraction of energy leaving one body that is intercepted by another (Mills 539). The shape factor for the radiation coming from the internal node to the external nodes is 1 because they are touching. The external nodes are absorbing all of the radiation emitted by the internal node since the dimensions of each side of the internal node are smaller than the side panel dimensions. The makes the shape factor for the radiation coming from the external nodes to the internal node the following fraction:

$$
F_{e i}=\frac{A_{\text {int }}}{A_{e x t}}
$$

In order to explain the shape factor equations for the external nodes to each other, Figure 4.4 displays a page from Mills (543).

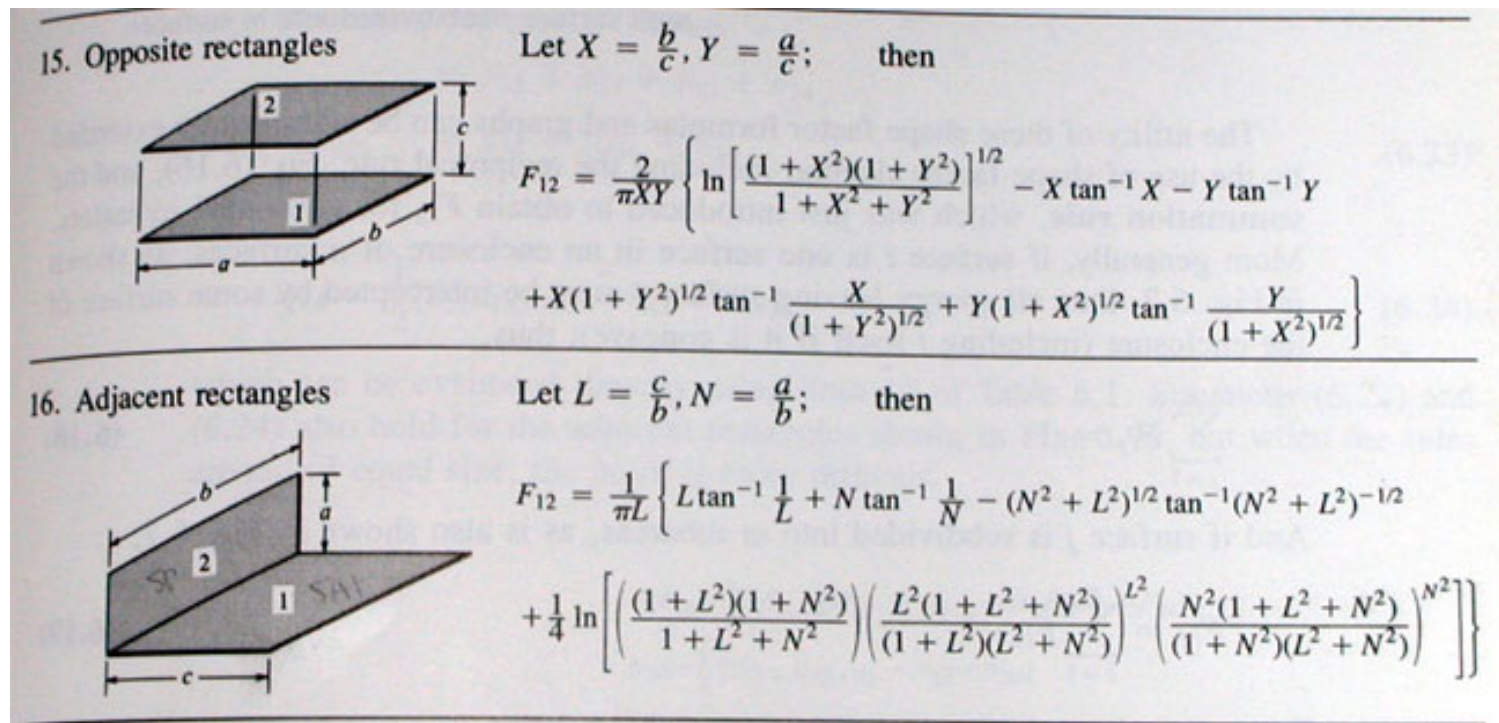

Figure 4.4. Shape Factor Equations for External Nodes

The dimensions used for the variables in Figure 4.4 are the dimensions of the CP3 side panels and their actual distances apart. Once the shape factors have been calculated, the 
radiosity $(\mathrm{J})$ of each node is found. Radiosity is "all the radiation that leaves the surface, whether emitted or reflected" (Mills 552). In order to calculate the radiosity of each node, the following system of equations has to be solved:

$$
\begin{gathered}
J_{i}=\varepsilon_{i} E_{b i}+\left(1-\varepsilon_{i}\right)\left[J_{1} F_{i 1}+J_{2} F_{i 2}+J_{3} F_{i 3}+J_{4} F_{i 4}+J_{5} F_{i 5}+J_{6} F_{i 6}\right] \\
i=1,2,3,4,5,6
\end{gathered}
$$

Using the radiosities, the radiation effect of all the external nodes on the internal node is:

$$
\dot{Q}_{A R}=F_{e i} A_{\text {int }} \sigma\left(J_{2}+J_{3}+J_{4}+J_{5}+J_{6}\right)
$$

The radiation effect of the internal node on one of the external nodes is:

$$
\dot{Q}_{A R}=A_{e x t} \sigma J_{1}
$$

\subsubsection{Using the Thermal Model for Verification}

The thermal model goes through two stages of calculations when used for verification of filter convergence. The first stage takes the actual side panel temperature data and uses it to calculate a temperature profile of the internal node. The second stage runs after the filter has finished and employs the SBEV and CBEV as inputs along with the internal temperature profile as initial conditions. The major correlations to look for between actual temperature data and the filtered data are the frequency of side panel temperature peaks and that the right side panels are in the sun at relatively the right time. The magnitude and timing of sunlit side panels will be slightly off since the model is not perfect.

\subsection{Filter Simulation}

\subsubsection{Satellite Emulator}

Once all the changes were made the PEKF, Sturms's Satellite Emulator was used once again to ensure that the PEKF is still working properly. To recap the logistics of the 
emulator, it numerically integrates the equations of motion to compile the "actual" states using a random initial state. The quaternion states are then used to rotate the MIRV into the body frame, creating the MBRV. The MBRV is "corrupted with white noise and given an 8-bit resolution of one Gauss to behave like the magnetometers as characterized" (Sturm 31). This magnetometer data is run through the PEKF to tune the parameters and test convergence. Internal node temperatures were added to the emulator, which are produced using a sine wave with the same frequency as the orbit being emulated and a magnitude of $15^{\circ} \mathrm{C}$.

\subsubsection{Emulated Filter Performance}

The PEKF was run with the initial $\mathrm{P}$ and $\mathrm{Q}$ parameters that gave the best performance in Erick's thesis. The simulation outputs the errors associated with the quaternions, rotation rates, side panel temperatures, and the magnetic field. Figure 4.5 displays the results from a simulation with 100 runs of the PEKF. The simulation demonstrated that the filter still works properly with a $100 \%$ convergence, taking between 1 orbit and 1.5 orbits.
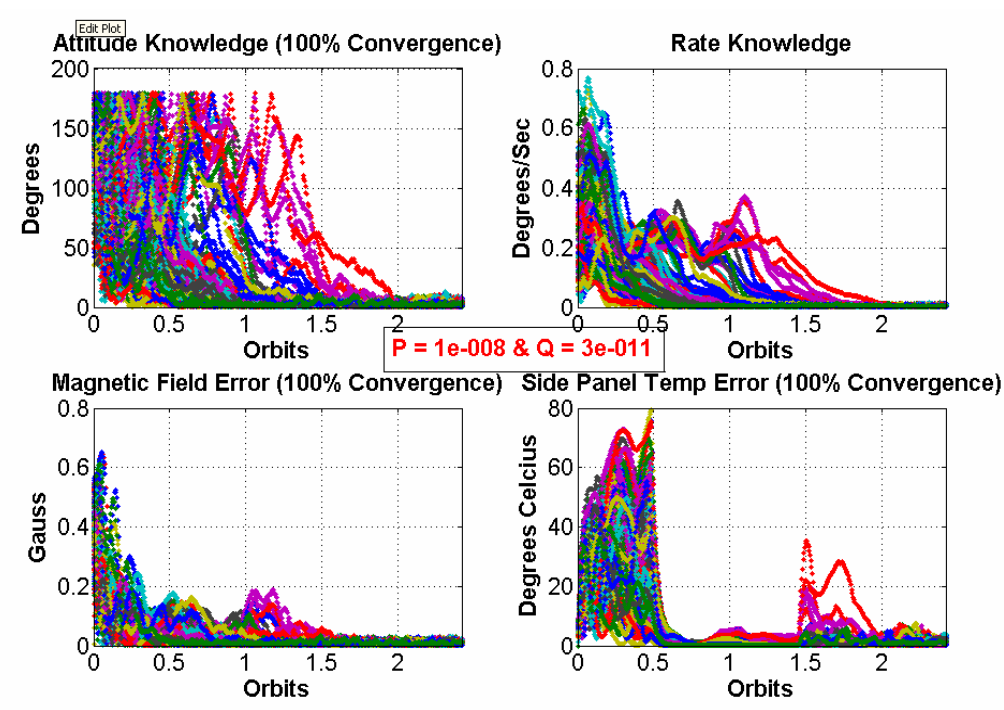

Figure 4.5. PEKF Simulation for Filter Testing 


\section{CHAPTER 5: PEKF PERFORMANCE WITH CP3 DATA}

\subsection{Filter Performance}

When the PEKF is run with data downloaded from CP3 while it's in orbit, four graphs are produced. The first graph contains two subplots: one with actual side panel temperature data and the other with the thermal model data using the filtered states. The temperature graph is used to verify that the filter is converging properly. The second graph displays the norm of the temperature error to show how well the model mimics the actual data and the performance of the filter. The third graph contains the norms of the MIRV's and the body frame magnetic field vectors that were transformed from the MIRV's using the filtered states. This graph checks the ephemeris data to demonstrate that the reference vectors were created for the correct points in space. The final graph presents the rotation rates in the body frame from the filter.

For this thesis, three data downloads were run through the filter: C\&DH data from July $9^{\text {th }}$ of 2007 , ADC data from July $12^{\text {th }}$ of 2007 , and ADC data from February $28^{\text {th }}$ of 2008 . These data packages were chosen due to their minimum amount of dropped packets. Having data that was six months apart also gave clues to the changes in the spacecrafts dynamics over time. The July $9^{\text {th }}$ data and the February $28^{\text {th }}$ data had a 30 second snapshot rate while the July $12^{\text {th }}$ data had a 10 second snapshot rate. The difference in snapshot rate was chosen to determine the affect that snapshot rate has on filter convergence. 


\subsection{Filter Convergence}

For the initial runs of the filter, the initial P and Q values that Erick Sturm found created the best results in the satellite emulator were used. This turned out to be insufficient for getting the PEKF to converge. An alternative method was developed where the temperature data was used to tune the filter's P and Q initial conditions by using trial and error until the filter converged properly. This became a tedious process due to the sensitivity of the covariance matrix to slight changes in its initial value.

\subsubsection{July $9^{\text {th }}, 2007$ Data}

The July $9^{\text {th }}$ of 2007 C\&DH data was download a little less than three months after launch. An assumption was made that the satellite still had a relatively high rotation rate from tip off out of the cubesat deployer. The magnetic field vector norms tracked perfectly, as shown in Figure 5.1. As stated earlier, this demonstrates that the time periods for the ephemeris data were chosen correctly and there were no anomalies with the data from the IGRF model. Figure 5.2 displays the side panel temperatures for this data. The figure contains the $\mathrm{P}$ and $\mathrm{Q}$ initialization used for this particular graph. The figure shows that the low frequency trends of the side panels match up relatively well despite the few spikes at the beginning while the filter is still converging. 


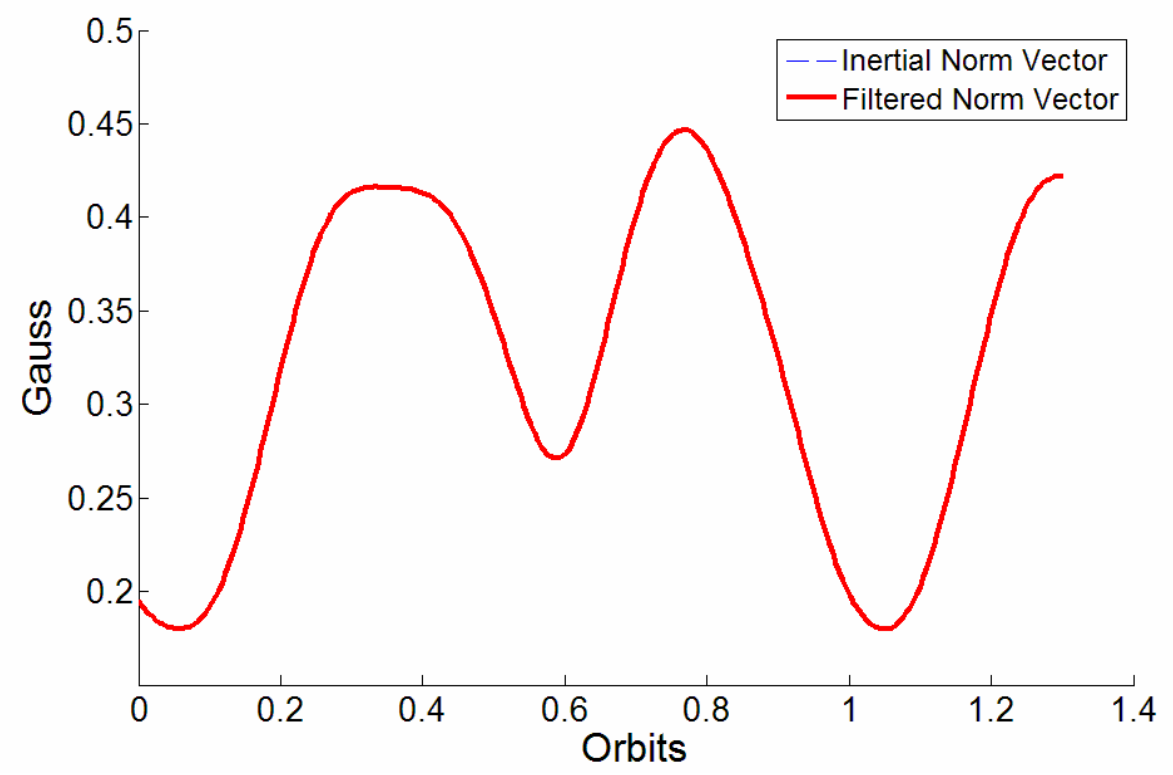

Figure 5.1. July $9^{\text {th }}, 2007$ Magnetic Field Vector Norms
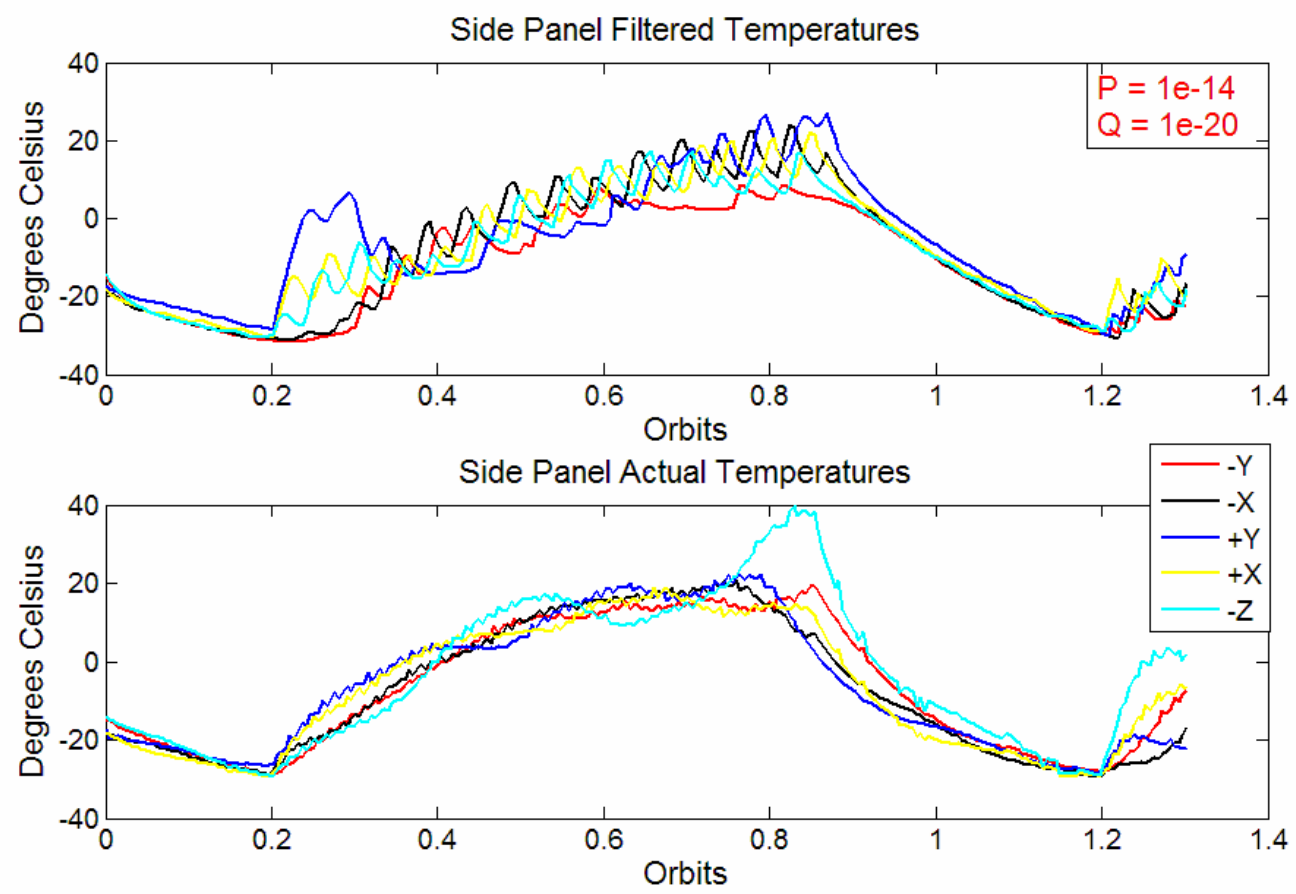

Figure 5.2. July $9^{\text {th }}, 2007$ Side Panel Temperatures 
The high frequency trends do not line up between the two subplots, which will be explained once the July $12^{\text {th }}$ data is shown. The difference between the two high frequency trends would suggest that the spacecraft is moving faster than the filter thinks it is. Figure 5.3 quantifies the error between the two temperature graphs by taking the norm of the error between the two sets of temperature data.

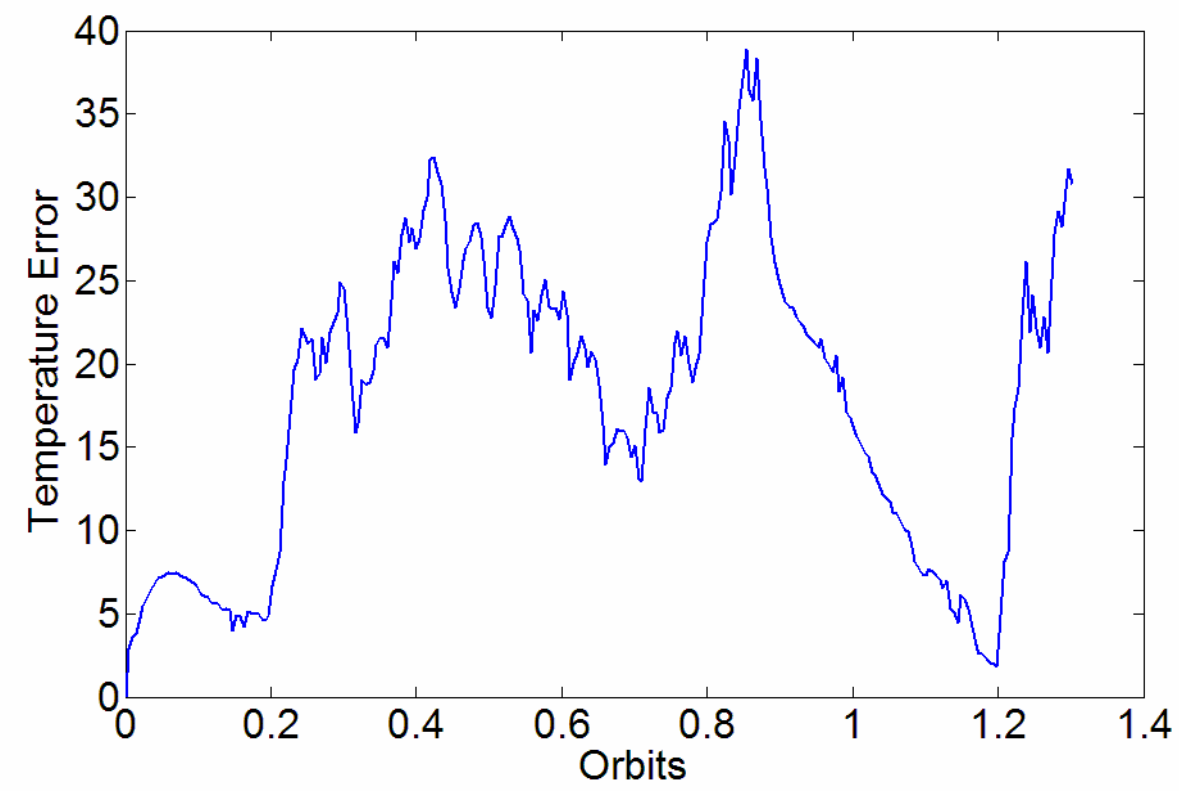

Figure 5.3 July $9^{\text {th }}$, 2007 Side Panel Temperature Error

Figure 5.3 illustrates both the error associated with the thermal model's ability to show the correct side panel lit by the Sun perfectly and how well it matches the magnitude of the temperatures at specific times. The high error for the thermal model comes from its inability to match the side panel temperature magnitude properly. For the July $9^{\text {th }}$ data, the error stems from the filter's failure to portray the high frequency temperature changes by not converging properly. 


\subsubsection{July $12^{\text {th }}, 2007$ Data}

The July $12^{\text {th }}$ data, which should closely resemble the July $9^{\text {th }}$ data, was taken with a 10 second sensor snap rate. The magnetic field vector norms for July $12^{\text {th }}$ are displayed in Figure 5.4. The two magnetic field vector norms line up perfectly, once again demonstrating a good choice for ephemeris data. The side panel temperatures are shown in Figure 5.5. In this case, the higher frequencies seem to be the same but the

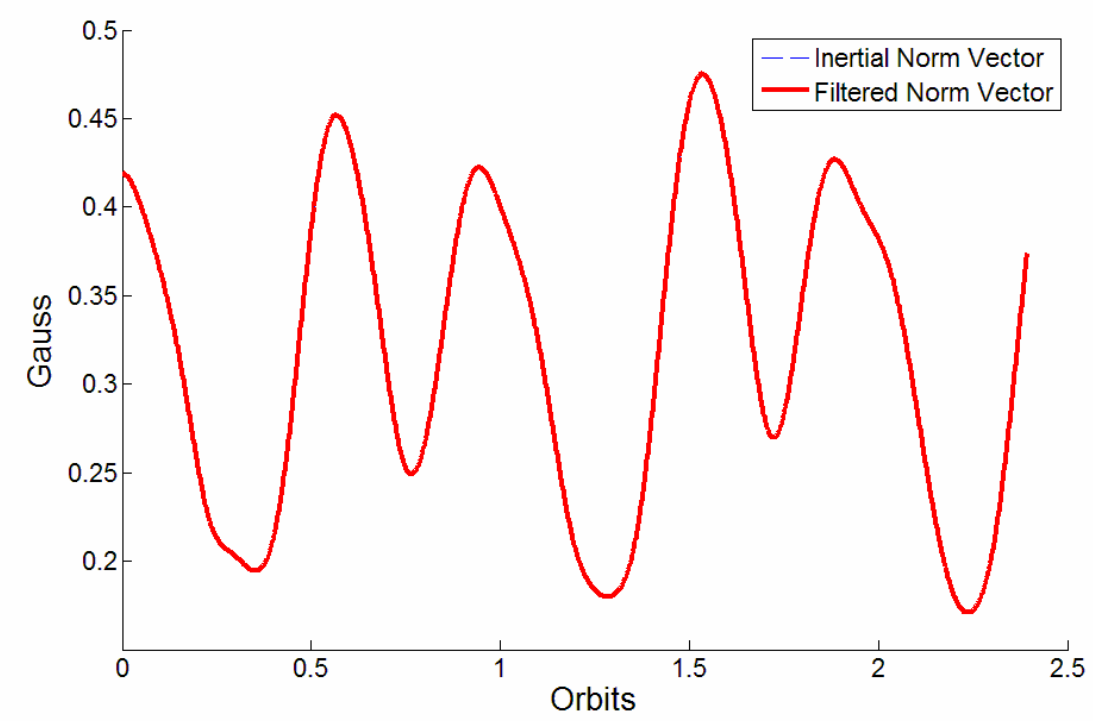

\section{Figure 5.4. July $12^{\text {th }}, 2007$ Magnetic Field Vector Norms}

lower frequencies do not match up as well. One cause of this could be the dropped packets in the data. The fit function used to fill in the dropped packets does not capture the high frequency data, which would throw off the filter during those segments. The satellite's last cycle demonstrates this event, where dropped packets stand out as sections of the data where the high frequency trends disappear. Figure 5.6 presents the side panel temperature errors. The errors were about the same for both the July $12^{\text {th }}$ data and the July $9^{\text {th }}$ data. Between 1.5 and 2.0 orbits, the error is slightly higher due to the fitted data from the dropped packets. Although the orientation information given by this filter run 

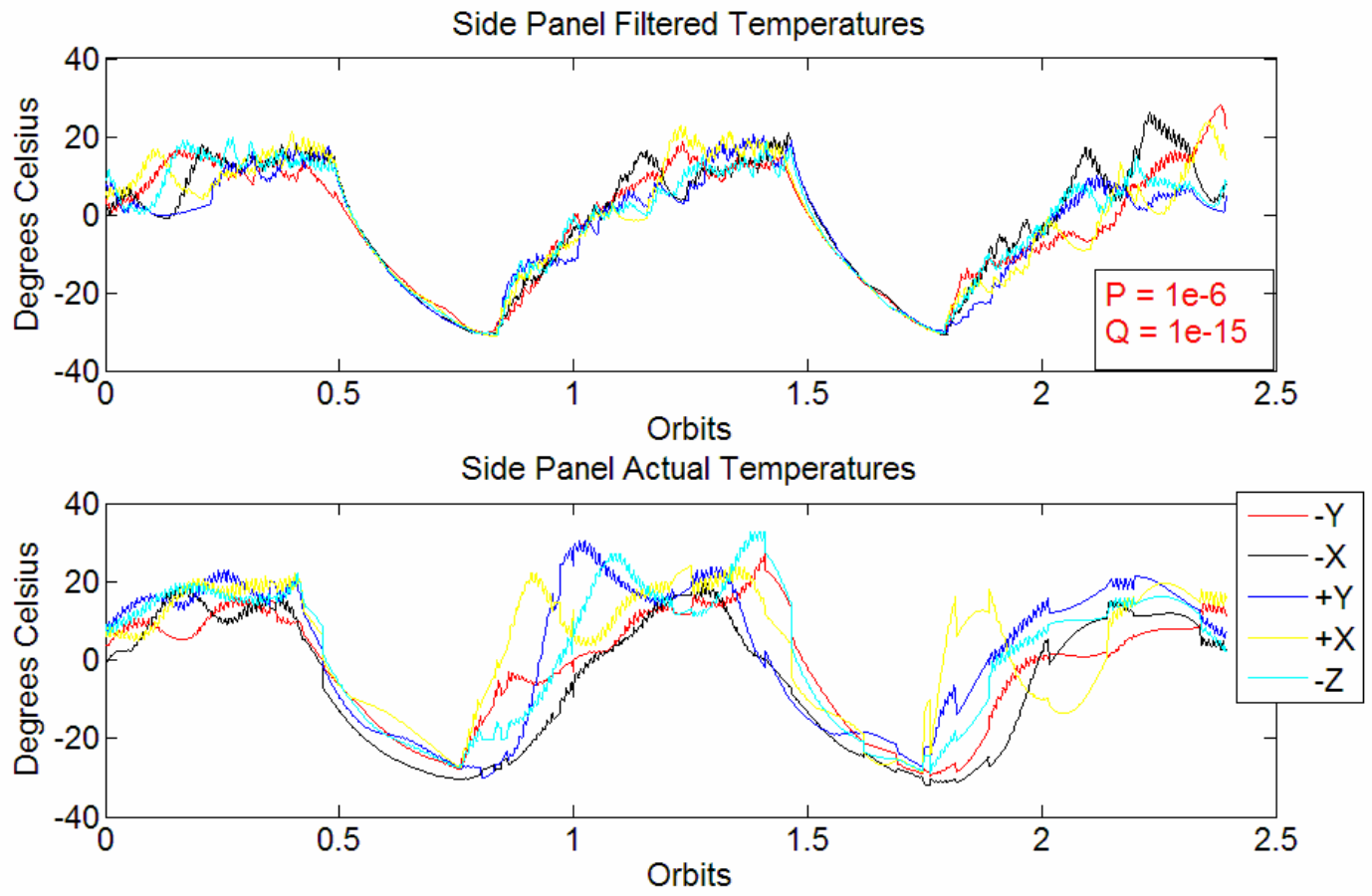

Figure 5.5. July $12^{\text {th }}, 2007$ Side Panel Temperatures

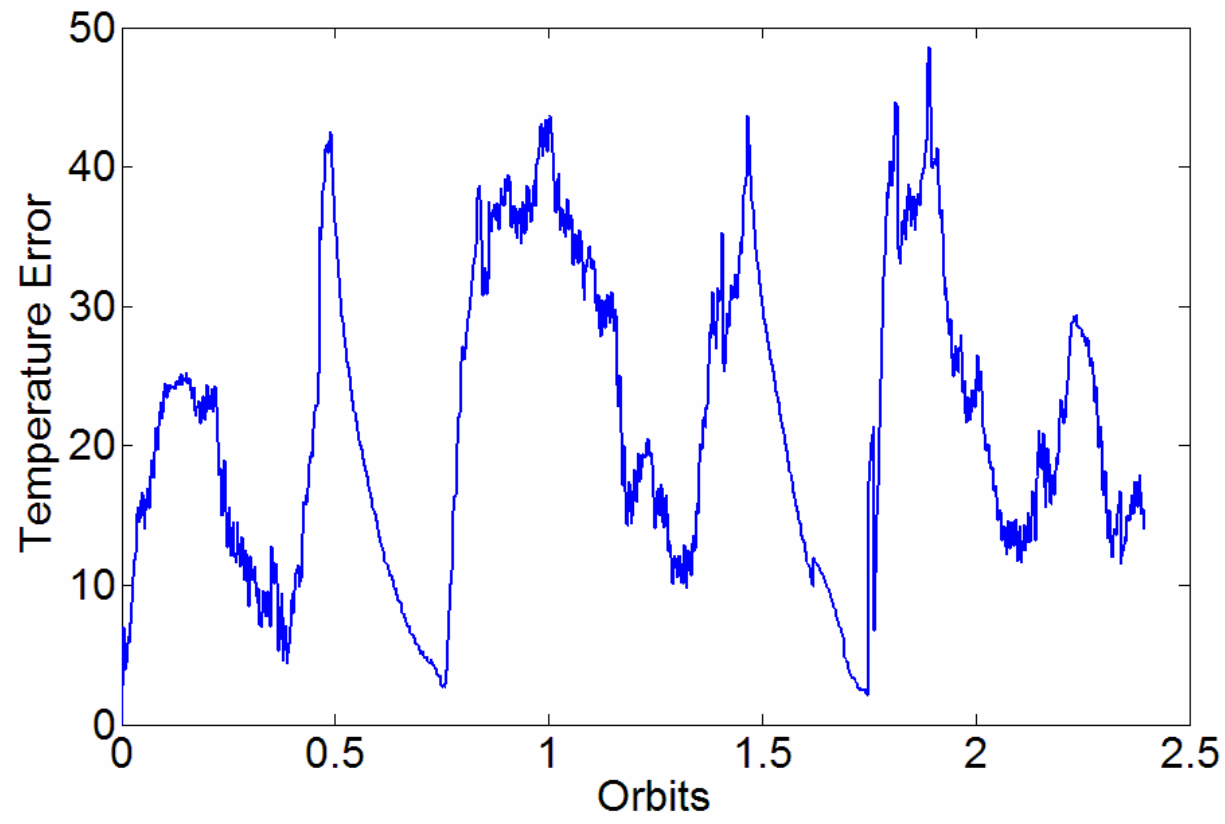

Figure 5.6 July $12^{\text {th }}, 2007$ Side Panel Temperature Error 
would be slightly inaccurate, the rotation rates would be more accurate than the July $9^{\text {th }}$ data. Since the July $9^{\text {th }}$ data was taken at a 30 second snap rate, it did not capture the magnitude of the high frequency trends. The filter probably accredited it to noise and filtered it out. One indication of this is the drastic difference in magnitude of the initial covariance matrices for the two runs. The July $9^{\text {th }}$ run had an initial covariance scalar much closer to zero than the July $12^{\text {th }}$ run. This signifies that the July $9^{\text {th }}$ run through the filter depended more on the spacecraft model rather than the data, where the July $12^{\text {th }}$ run was the opposite.

\subsubsection{February $28^{\text {th }}, 2008$ Data}

The February $28^{\text {th }}$ data also had a 30 second snapshot rate yet it occurred 6 months after the other two data downloads. One thing that sets this data apart from the others is that there are no dropped packets. Figure 5.7 presents the magnetic field vector norms. Once again, the two norms are a perfect match. The side panel temperatures are displayed in Figure 5.8. The actual side panel data does not contain any high frequency trends,

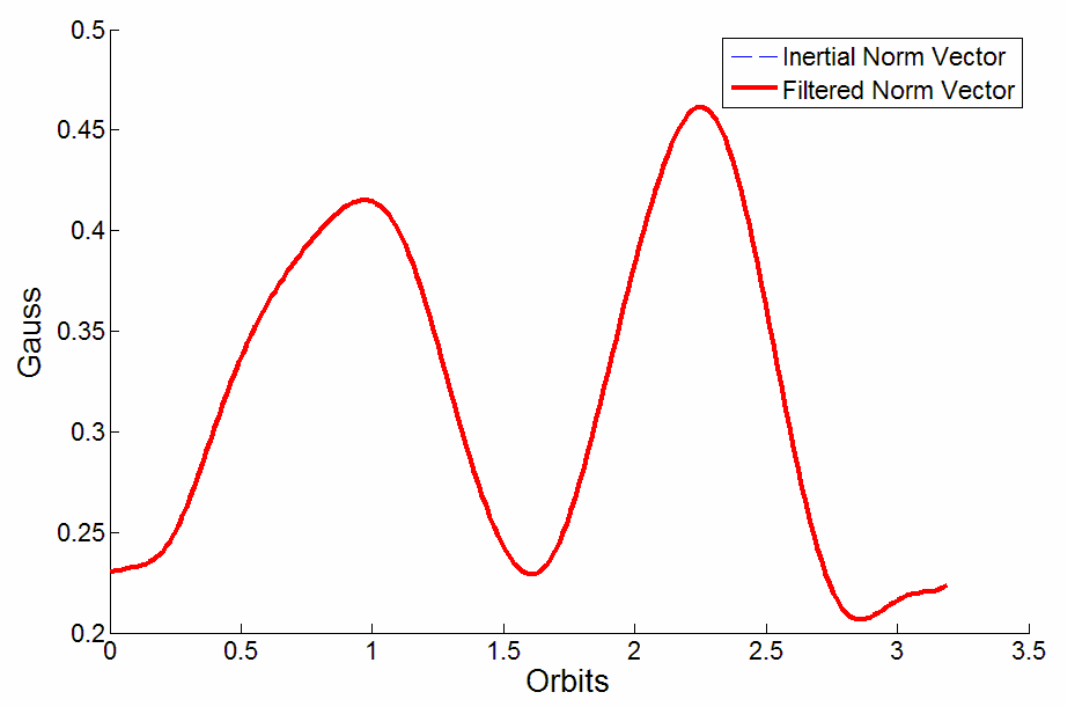

Figure 5.7. February $28^{\text {th }}$, 2008 Magnetic Field Vector Norms 

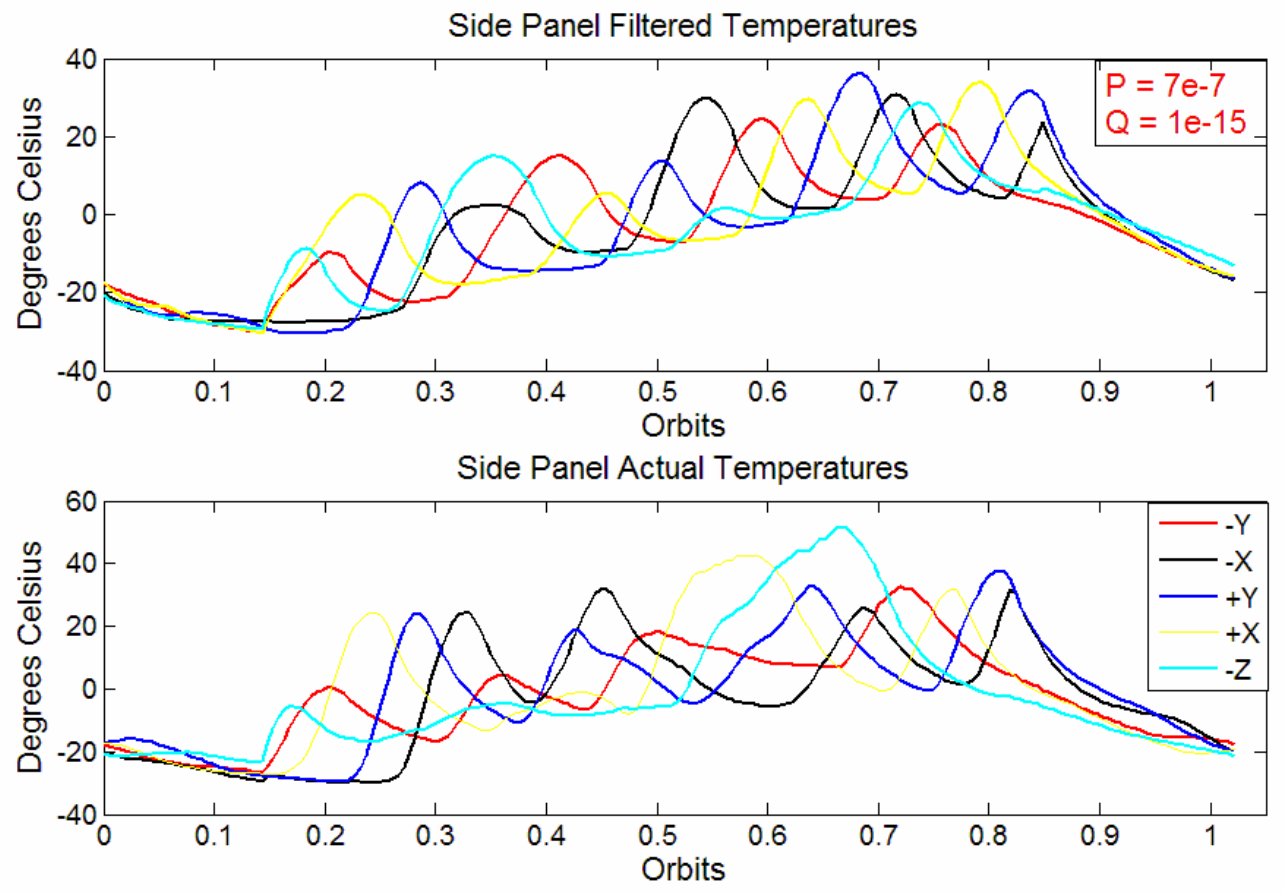

Figure 5.8. February $28^{\text {th }}, 2008$ Side Panel Temperatures

indicating that the satellite's rotation rate slowed down over the six-month period. The initial covariance scalar has a value close to the July $12^{\text {th }}$ s covariance scalar, showing that this filter run relies more on the data than the satellite model. Figure 5.9 shows the side panel temperature errors for this data set. The error is essentially the same as the other runs, except during 0.4 and 0.8 orbits, where it seems there is an extra rotation based on Figure 5.8. This indicates that the 30-second snapshot rate is sufficient for slower rotation rates, although there is still some error associated to the filter. Some of the error comes from the already mentioned problem with the magnitude of the temperature model. 


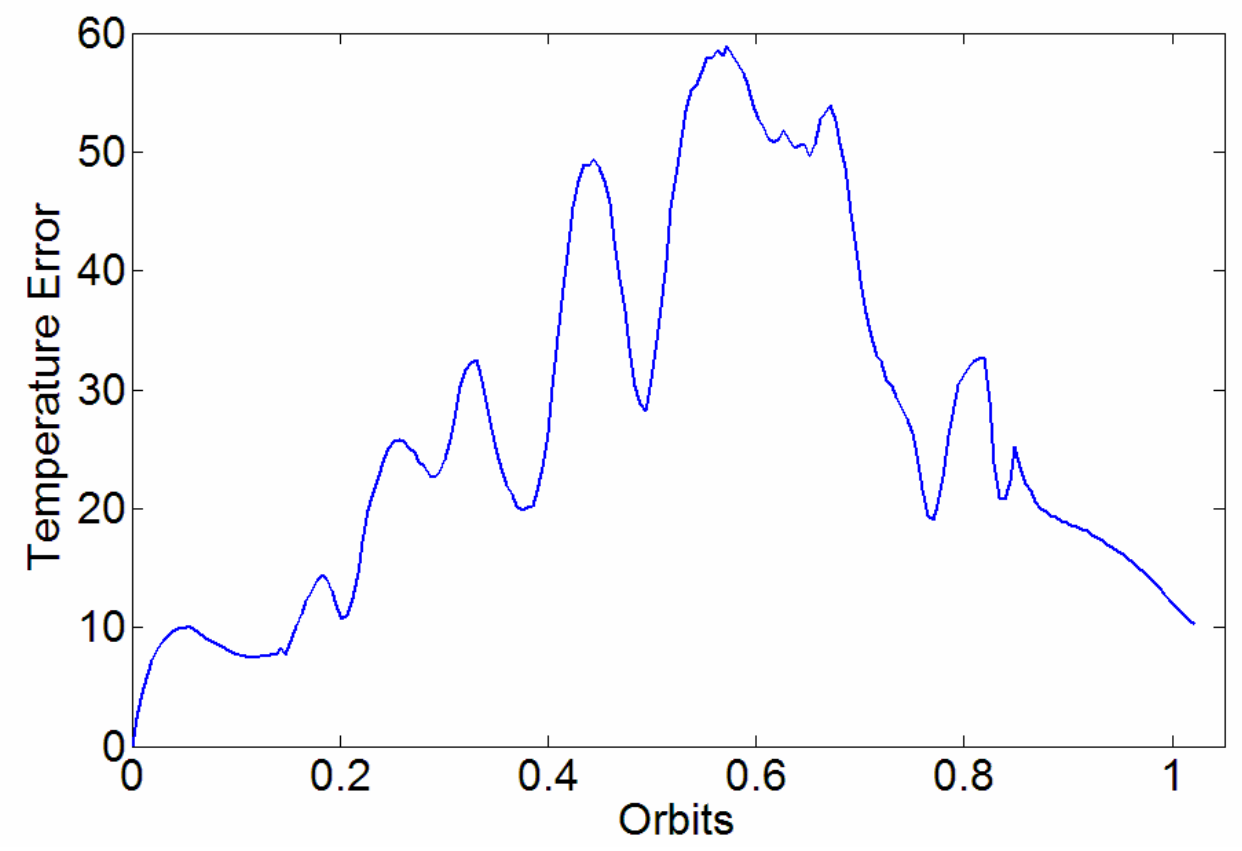

Figure 5.9. February $28^{\text {th }}, 2008$ Side Panel Temperature Error

\subsection{Solar Panel Current Issues}

According to Humphrey, the solar panels could be used as crude sun sensors using solar panel power (1). Sturm included a convergence verification tool in his original PEKF using the filtered Sun vector in the body frame to generate solar panel power that could be compared with actual solar panel power using the current sensors. While analyzing the first sets of data from CP3, two issues were found with the solar panel current readings. The first issue concerned the solar panel current sensors, which were railing during large incidence angles of the Sun vector on the solar cells. The second issue had to due with the uplink problems with the satellite that did not allow it to run at full power. Since the batteries were not being utilized, they would recharge quickly after the satellite came into the Sun's view. This caused the current draw from the solar panels to be intermittent, causing spikes in the data. Figure 5.10 shows the normalized solar panel 
power from the solar panel current readings running off 4.7 volts along with the predicted solar panel power from the filter using a cosine cubed relationship.
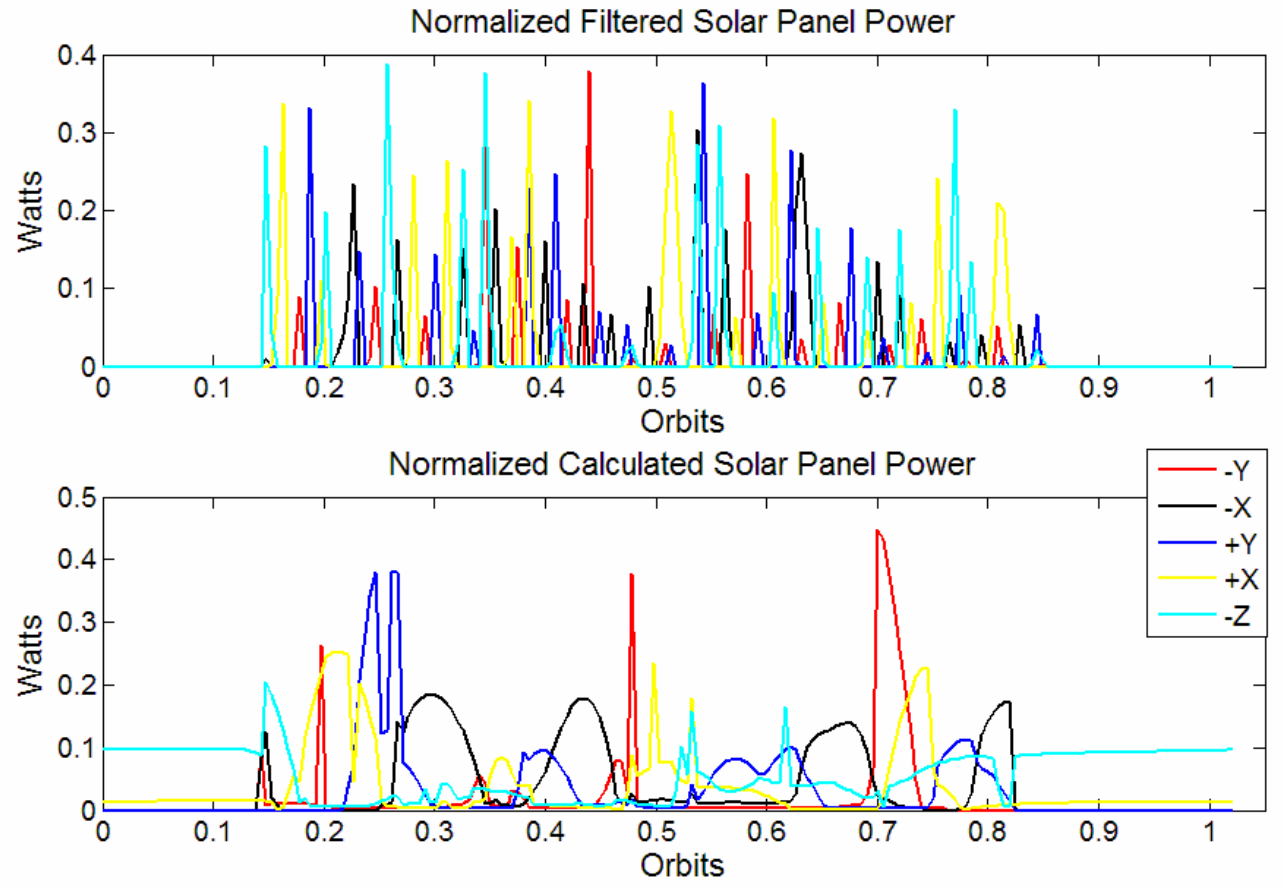

Figure 5.10. February $28^{\text {th }}, 2008$ Normalized Solar Panel Power

One thing to note from the figure are the $-\mathrm{Z}$ and $+\mathrm{X}$ solar panel current sensor malfunctioned and gave current readings even when the satellite was not in the Sun. This shows that the solar panels can only be used as crude sun sensors if the power budget is designed so that the batteries are never running completely full while the satellite is in the Sun. 


\section{4 Rotation Rates}

Rotation rate trends reveal the change in the spacecraft dynamics over time. Figure 5.11 and Figure 5.12 display the rotation rates for July $9^{\text {th }}$ of 2007 data and July $12^{\text {th }}$ of 2007 data, respectively.
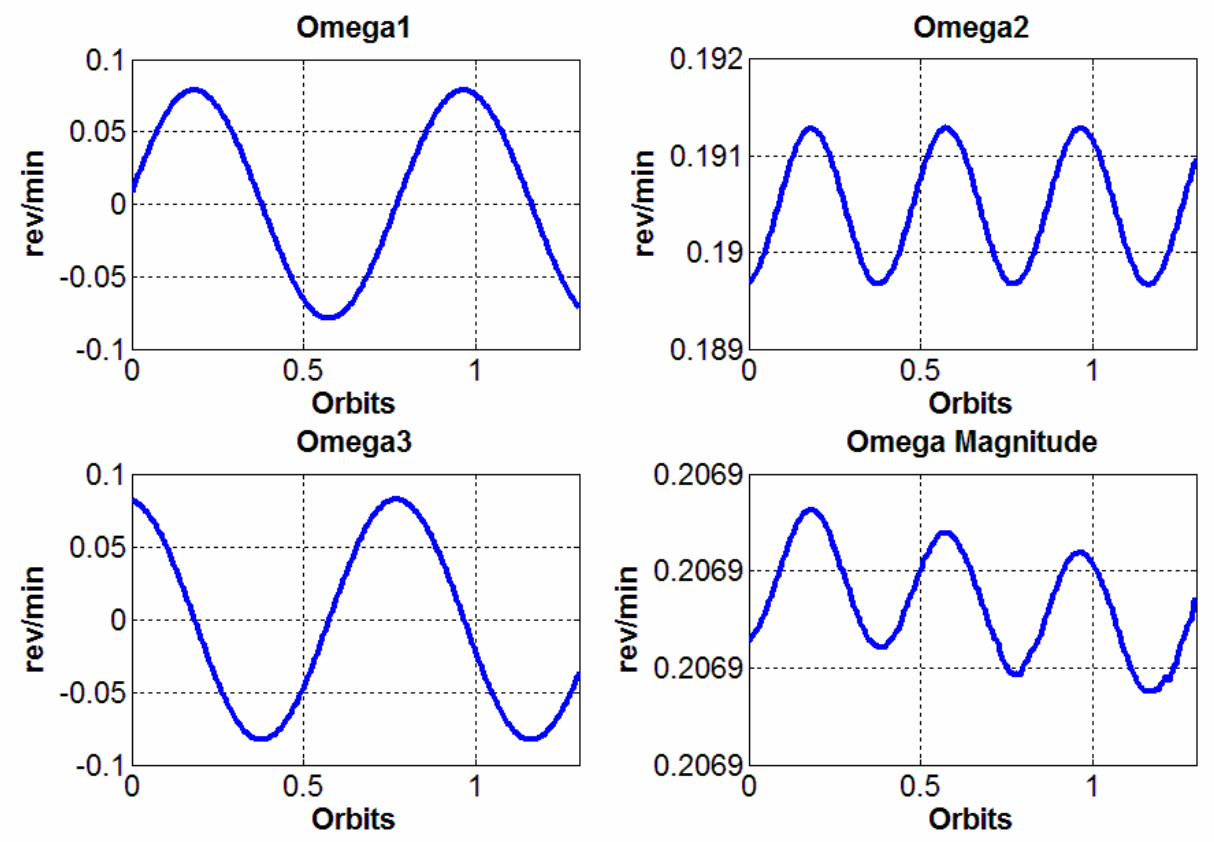

Figure 5.11. July $9^{\text {th }}$, 2007 Rotation Rates 

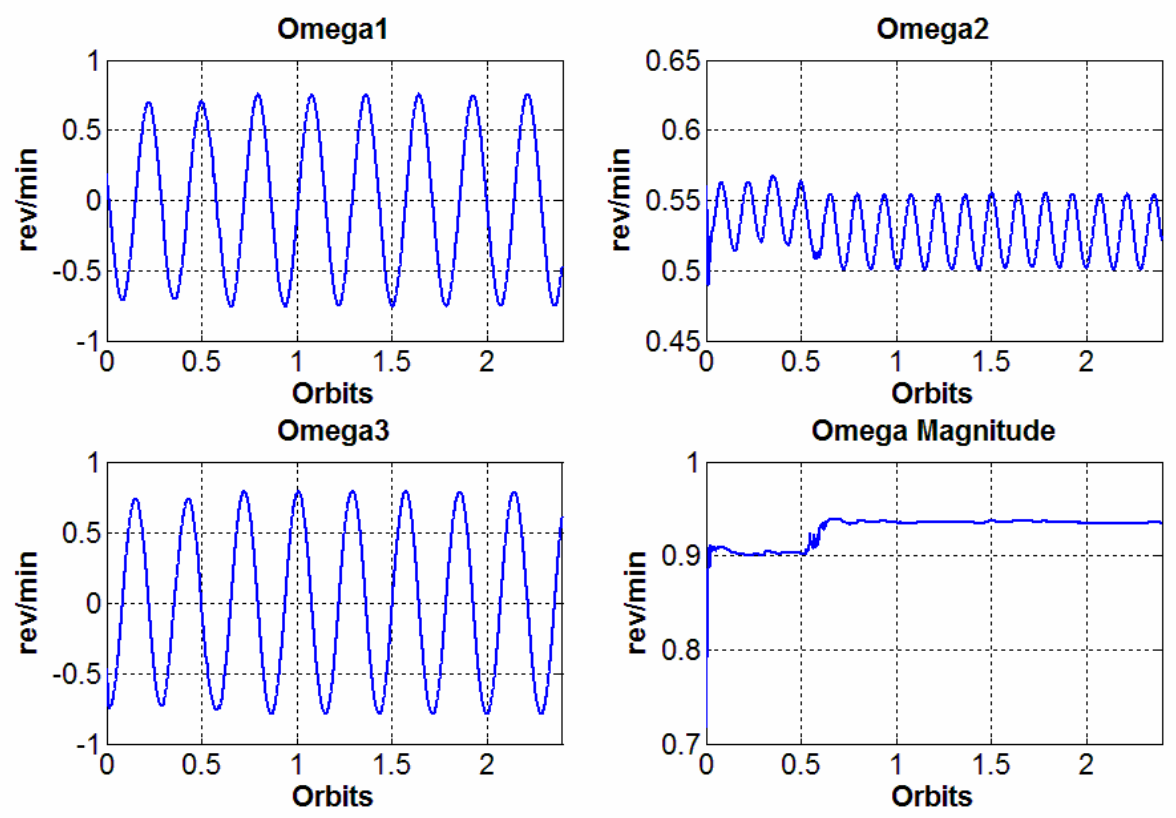

Figure 5.12. July $12^{\text {th }}, 2007$ Rotation Rates

The designators 1,2 , and 3 in the rotation rate figures refer to the body axes $\mathrm{x}, \mathrm{y}$, and $\mathrm{z}$. These two figures demonstrate the affect that snapshot rate has on data from a satellite with a fast rotation rate. The sinusoidal movement of $\omega_{1}$ and $\omega_{3}$ between positive and negative values indicates that the satellite had a nutation while it was rotating about the $\omega_{2}$ axis. Both figures indicate this, showing that it was most likely the trend of the satellite. The July $12^{\text {th }}$ data presents a much faster rotation rate, due to its reliance on the higher resolution data. For the July $9^{\text {th }}$ data, the filter captured the basic movement of the satellite relying more on the model of the system. Figure 5.13 presents the rotation rates for the February $28^{\text {th }}$ of 2008 data. 

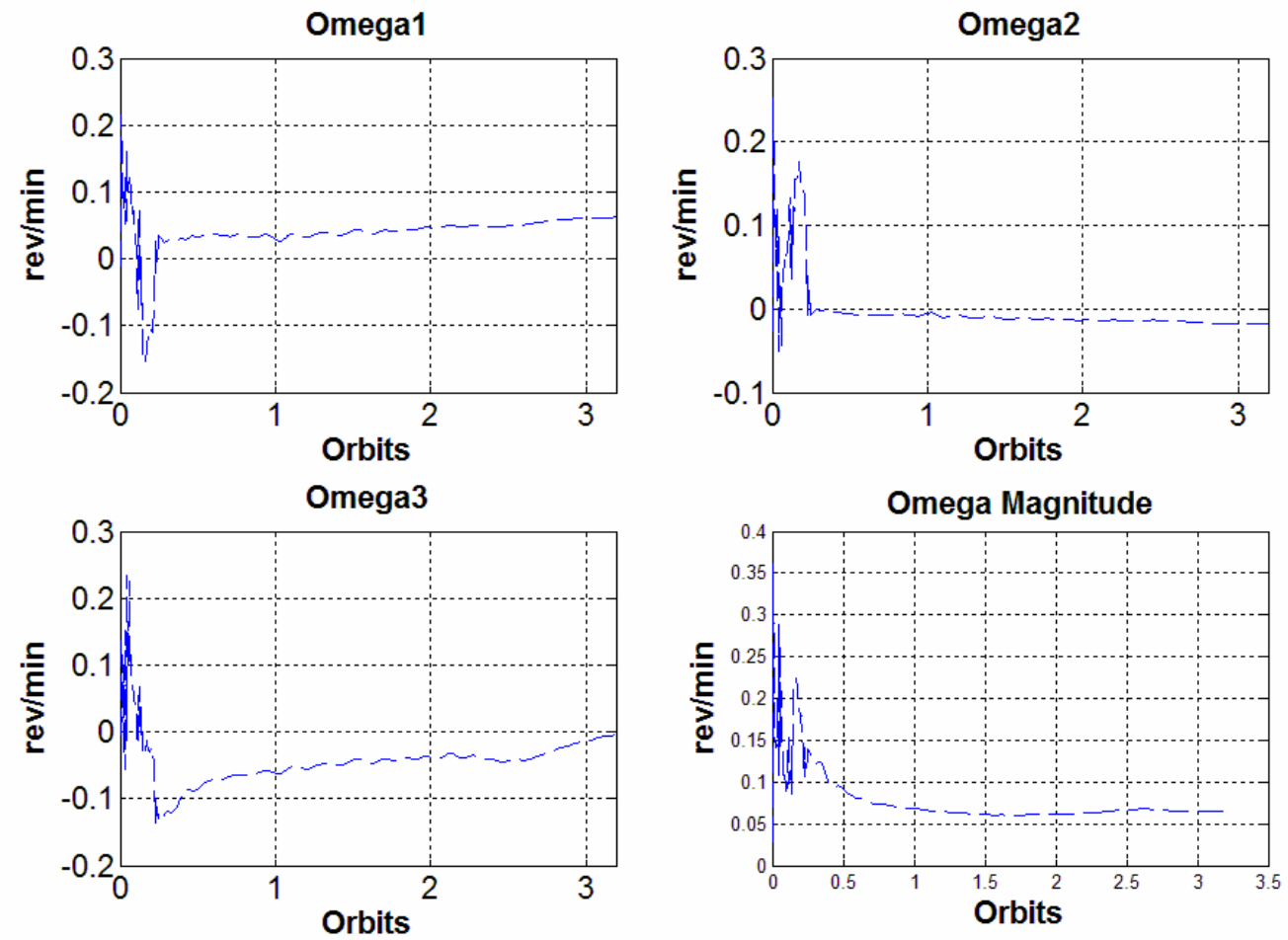

Figure 5.13. February $28^{\text {th }}, 2008$ Rotation Rates

Figure 5.13 shows filter convergence in a little less than half of an orbit. This was confirmed by the thermal data from section 5.1. When compared with the July $12^{\text {th }}$ data, the rotation rate of the satellite drastically slowed down in six months. One theory on this slowing in rotation rate is that the antenna is a source of magnetic field onboard the spacecraft, which is trying to align itself with Earth's magnetic field.

\subsection{Magnetic Field Alignment}

In order to test this theory of a magnetized antenna, the magnetometer data is plotted in the body frame with the position of the dipole antennas indicated. The magnetometer data is taken from the February $28^{\text {th }}$ data set, since the rotation rate of the spacecraft had time to stabilize after launch. Figure 5.14 shows the February $28^{\text {th }}$ of 2008 magnetometer 
data in the body frame of the satellite. The figure gives compelling evidence that the antenna is magnetized, causing it to attempt to align itself with the Earth's magnetic field. It would seem that the satellite has magnetic alignment without it being designed into the system.

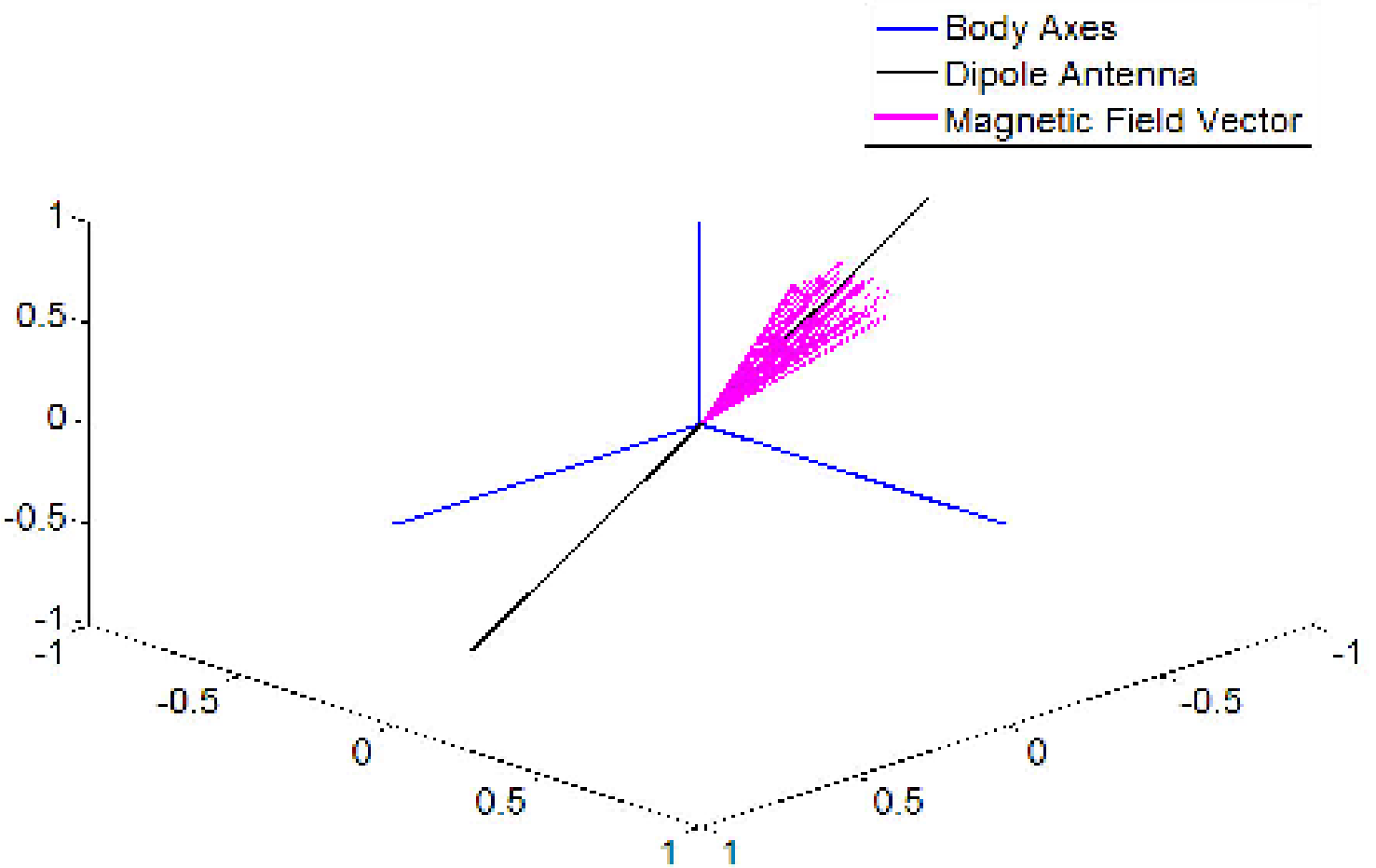

Figure 5.14. Magnetic Field Vectors in the Body Frame 


\section{CHAPTER 6: CONCLUSION}

\subsection{Summary}

The modified PEKF runs with actual on-orbit data downloaded from CP3. The thermal model built for the PEKF is used to tune the initial parameters of the filter as well as convergence verification. It is accurate enough to capture the frequency of temperature changes and generally the correct side panel being lit by the sun if the filter converges properly. The thermal model has trouble representing the correct magnitude of the temperature on a side panel. Dropped packets play a large role in filter divergence and accuracy, even with a curve fit of the data. Downloaded data snapshot rates also affect filter divergence and accuracy. A 30-second snapshot rate is sufficient for slower rotation rates; yet 10-second snapshots are necessary for faster rotation rates.

Quantifying what a "slower" or "faster" rotation rate would take more analysis. In this case, a 30 second snapshot rate made the filter converge to more accurate attitude information for a $0.1 \mathrm{rev} / \mathrm{min}$ rotation rate than $1.0 \mathrm{rev} / \mathrm{min}$ rotation rate. There were some problems with inaccurate filtered states even with filter convergence, which could stem from unknown moment of inertia values propagating within the states. The ability of the solar panels to work as crude sun sensors was analyzed using filtered Sun vector information and side panel current data. The solar panel current cannot be used as crude sun sensors because of insufficient current draw and the railing of current sensors. By investigating the drastic reduction in the CP3's rotation rate over 6 months, it was discovered that the satellite's antenna is most likely magnetized, causing it to try to align itself with the Earth's magnetic field, slowing down the rotation rate of the satellite. 


\subsection{Recommendations for Future Work}

The thermal model needs work to improve its accuracy. More nodes could be added to improve the representation of the satellite in the model. With a more accurate thermal model, the side panel temperatures can be added to the measurements of the PEKF. They would work like a crude sun sensor.

The orbit propagator that was started by Erick Sturm in his thesis could be modified to reduce the complex computations necessary to run it. With a reduced orbit propagator that only needs to be updated at a reasonable interval, it could be programmed onboard the spacecraft. With an onboard orbit propagator, an onboard filtering program could be implemented.

The moments of inertia of the satellite could play a large role in the divergence of the PEKF. The current moments of inertia were calculated using a solid modeling program with a model of the spacecraft. This introduces unknown errors into the system, which could have a large impact on the filter. Simulations could be developed that found the effect of these errors in the moments of inertia of the satellite. Another filter could be developed that estimated the moments of inertia of the spacecraft while the PEKF is running, such as a dual extended Kalman filter or a joint extended Kalman filter.

Once the filter convergences consistently, an algorithm could be developed that ran the same data forward and backwards multiple times. This would smooth out the noise present at the beginning of the run before the filter converged completely. It would also dampen the noise present throughout data that was not reduced on the first pass. 


\section{LIST OF REFERENCES}

Astronomical Almanac for 2000 U.S. G.P.O.

Barreiro, Diego A. Melani, “A Small Satellite Preliminary Thermal Control and Heat Shield Analysis", Massachusetts Institute of Technology, December 2006.

Brown, R.G., and Hwang, P.Y.C., Introduction to Random Signals and Applied Kalman Filtering $3^{\text {rd }}$ Ed. New York: John Wiley \& Sons, Inc., 1997.

Challa, M., Natanson, G., and Wheeler, C., "Simultaneous Determination of Spacecraft Attitude and Rates Using Only a Magnetometer", AIAA paper, AIAA-96-3630-CP

Chen, H.C., C.C. Wan, Y.Y. Wang, "Thermal analysis of lithium-ion batteries.” Journal of Power Sources 140 (2005): 111-124. 29 Sept. 2004

$<\mathrm{http}: / /$ www.elsevier.com/locate/jpowsour $>$

Humphreys, T.E., “Attitude Determination for Small Satellites with Modest Pointing Contraints", Utah State University, Small Sat, 2002.

Krogh, K., "Attitude Determination for the AAU CubeSat", Aalborg University, June 2002.

Kuipers, Jack B., Quaternions and Roation Sequences. Princeton: Princeton UP, 2002.

Le, Huong G. and Dudely L. O’Brien, Process for Producing a High Emmitance Coating and Resulting Article. U.S. Patent No. 5,217,600: Issued 1993.

Lefferts, E.J., Markley, F.L., and Shuster, M.D., "Kalman Filtering for Spacecraft Attitude Estimation", Journal of Guidance, Control, and Dynamics, Vol. 5, No. 5, 1982, pp. 417-429.

Mills, A.F., Heat Transfer. Upper Saddle River: Prentice Hall, 1999.

Psiaki, M.L., Martel, F., and Pal, P.K., "Three-Axis Attitude Determination via Kalman 
Filtering of Magnetometer Data", Journal of Guidance, Control, and Dynamics, Vol. 13, No. 3, 1989, pp. 506-514.

Shabany, Younes, "Component Size and Effective Thermal Conductivity of Printed Circuit Boards", The Eighth Intersociety Conference on Thermal and Thermomechanical Phenomena in Electronic Systems (2002): 489-494.

Spectrolab. 28.3\% Ultra Triple Junction (UJT) Solar Cells. 29 May 2009 $<$ http://www.spectrolab.com/DataSheets/TNJCel1/utj3.pdf $>$

Sturm, Erick Jonathan, "Magnetic Attitude Estimation of a Tumbling Spacecraft", California Polytechnic State University, July 2005.

Svartveit, K., "Attitude Determination of the NCUBE Satellite", NTNU, June 2003. Wertz, J.R., (ed.), Spacecraft Attitude Determination and Control, Kluwer, 2005. 